\title{
Gauge fields and infinite chains of dualities
}

\author{
Nicolas Boulanger, ${ }^{a}$ Per Sundell ${ }^{b}$ and Peter West ${ }^{c}$ \\ ${ }^{a}$ Service de Mécanique et Gravitation, Université de Mons - UMONS, \\ 20 place du Parc, B-7000 Mons, Belgium \\ ${ }^{b}$ Departamento de Ciencias Físicas, Universidad Andres Bello - UNAB, \\ Av. República 252, Santiago, Chile \\ ${ }^{c}$ Department of Mathematics, King's College, \\ London WC2R 2LS, U.K. \\ E-mail: nicolas.boulanger@umons.ac.be, per.sundell@unab.cl, \\ peter.west@kcl.ac.uk
}

ABstract: We show that the particle states of Maxwell's theory, in $D$ dimensions, can be represented in an infinite number of ways by using different gauge fields. Using this result we formulate the dynamics in terms of an infinite set of duality relations which are first order in space-time derivatives. We derive a similar result for the three form in eleven dimensions where such a possibility was first observed in the context of $E_{11}$. We also give an action formulation for some of the gauge fields. In this paper we give a pedagogical account of the Lorentz and gauge covariant formulation of the irreducible representations of the Poincaré group, used previously in higher spin theories, as this plays a key role in our constructions. It is clear that our results can be generalised to any particle.

Keywords: Duality in Gauge Field Theories, Gauge Symmetry, M-Theory

ArXiv EPrint: 1502.07909 


\section{Contents}

1 Introduction 1

2 Spin one and its gauge fields 3

2.1 The Wigner unitary irreducible representation of spin one 3

$\begin{array}{ll}2.2 & \text { Dualities and Gauge potentials }\end{array}$

2.3 Action principle for the dual potential $A_{c[D-2] \| a}^{(1)} \quad 15$

3 The three form in eleven dimensions $\quad 17$

$\begin{array}{ll}3.1 \text { The unfolded representation of the three-form } & 17\end{array}$

$\begin{array}{lll}3.2 & \text { Further dualisation of the three form } & 23\end{array}$

3.3 Unfolded description containing the $A_{[9,3]}$ form 26

3.4 First-order frame-like action for the $A_{[9,3]}$ field 29

3.5 Higher dualisations on-shell 31

4 Discussion $\quad 33$

$\begin{array}{ll}\text { A Notation } & 34\end{array}$

\section{Introduction}

Dirac's wish to treat the electric and magnetic fields of Maxwell's equation in a more symmetric fashion lead him to propose the existence of magnetic monopoles [1]. These were found to occur as regular solutions of spontaneously broken Yang-Mills theories coupled to scalar fields [2, 3]. However, it was Montonen and Olive who proposed that there might be an electromagnetic duality symmetry [4] which was subsequently found to be present in the maximally rigid supersymmetric theory. One of the most important discoveries in supergravity theories was the existence of exceptional symmetries in the maximally supergravity theories [5]. The scalar fields in these theories belong to a coset space constructed from the exceptional symmetry. However, these symmetries generically act on the other fields in the supergravity theory and when acting on the "spin one" fields they act as a kind of electro-magnetic duality symmetry [6].

It has been conjectured that the underlying theory of strings and branes possess a very large Kac-Moody symmetry called $E_{11}$ [7]. This is encoded in a non-linear realisation which possesses an infinite number of fields. The fields are ordered by a level and at low levels the fields in $E_{11}$ are just those of maximal supergravity theory in the dimension being considered. However the $E_{11}$ theory is democratic in that it also contains the dual fields as well as the traditional fields; for example, in eleven dimensions in addition to the graviton and three form, it contains the six form and a field with the index structure $h_{a_{1} \ldots a_{8}, b}$ which was the dual of the gravity [7]. Indeed, it was in this paper that an equation of motion in $D$ 
dimensions of a field with the index structure $h_{a_{1} \ldots a_{D-3}, b}$ and the usual graviton was given at the linearised level. As this equation was derived form the usual formulation of gravity it was guaranteed to describe gravity in the correct way including the correct degrees of freedom. In fact the equation of motion of the dual graviton had been previously given in five dimensions in reference [8] where it was also pointed out that it had the correct degree of freedom to be gravity. The form of the dual graviton field had also previously been suggested in [9]. Finally, in [10] the action and complete set of gauge symmetries for the dual graviton in arbitrary dimension was constructed along the lines of [7], thereby tying together the results of [7] and [8].

Although, the fields in the $E_{11}$ non-linear realisation are listed for low levels, see for example [11], they are not systematically known at higher levels theory. However, certain results are known, these include all the $p$-form fields $[12,13]$ in the different dimensions, some of which play a key role in gauged supergravities. Also known are all fields that do not have blocks of ten and eleven indices in eleven dimensions [14]. These fields have a particularly simple form, they are just the usual fields of the maximal supergravity theory, as well as the dual fields just discussed above, as well as an infinite number of fields that consist of adding blocks of 9 indices to these fields. In eleven dimensions these are the fields

$$
\left\{h_{[1,1]}, A_{[3]}, A_{[6]}, h_{[8,1]}, A_{[9,3]}, A_{[9,6]}, h_{[9,8,1]}, A_{[9,9,3]}, A_{[9,9,6]}, h_{[9,9,8,1]}, \ldots\right\}
$$

where the numbers in square brackets indicate the number of antisymmetrised indices in each block. It was noted that when decomposed to the little group $\mathrm{SO}(9)$ these blocks of nine indices did not transform and so these fields should be just alternative ways of describing the degrees of freedom given in terms of the three form and graviton. In the sector of the graviton, this conjecture was verified in [16] at the action level. As a result $E_{11}$ encodes an infinite duality symmetry which should be expressed through an infinite series of duality relations which determine the dynamics of the particle. The analogous results for other very extended algebras were given in [15].

In this paper we will show that alternative gauge field representations arise quite generally for any particle. Indeed, they arise naturally from the irreducible unitary representations of the Poincaré group $\operatorname{ISO}(1, D-1)$ that describe any particle moving in Minkowski space-time. These are constructed following the method of Wigner, which involves the induced representation based on the isotropy, or little, group that preserves the momentum in a chosen Lorentz frame [17, 18]. In the massless case, this method works with the gauge field and as a result it has a number of ad hoc steps associated with the gauge transformation of this field.

However, there is another type of representation of $\operatorname{ISO}(1, D-1)$ also capable of carrying the massless, irreducible and unitary representations of $\operatorname{ISO}(1, D-1)$, and that is manifestly Lorentz covariant and also gauge invariant [19-23], see also [24-26]. This works with the fields strengths and their derivatives rather than the gauge fields. We note also the anterior and different method [27, 28] where field equations for arbitrary gauge fields are also formulated in terms of curvature tensors. When viewed in this way we will show that there is an infinite number of ways of introducing different gauge potentials corresponding to the particular equations one takes to be Bianchi identities and those one considers to 
be equations of motion. This choice reflects the possibility of the different possible duality transformations one can carry out on the field strengths and all their space-time derivatives. In the case of the spin-2 gauge field, this mechanism was explained in reference [16] using the fields contained in the $E_{11}$ non-linear realisation [7].

In this paper we will carry out this programme for Maxwell's theory in $D$ dimensions and then for the three form in eleven dimensions. As is very well known the latter occurs in the eleven dimensional supergravity theory [5]. We will find the equations of motion for the particles when described in terms of any of the possible gauge fields. We will also find an infinite series of duality relations that encode the dynamics of the particles and involve all the gauge potentials.

For the first sections of the paper we use familiar conventions for writing indices on fields, but as the paper progresses, and the number of different types of indices increases, we use a number of shorthand conventions. We define these as we use them, but for easy reference we give an appendix where these conventions are listed.

\section{Spin one and its gauge fields}

In this section we illustrate the ideas of this paper in the context of the simplest model, that is, the Maxwell theory in $D$ dimensions. We will show that this system possesses an infinite number of descriptions corresponding to an infinite number of different possible choices of gauge fields. The states of any particle are the irreducible unitary representation of the Poincaré group $\operatorname{ISO}(1, D-1)$ in $D$ dimensions. As we mentioned above, these were first found by Wigner [17] who constructed them as an induced representation of $\mathrm{ISO}(1, D-1)$ with respect to an isotropy subgroup that preserves a fixed momentum and it is this formulation that is most widely known. The representations are labelled by the representations of the isotropy group that they carry. In the massless helicity cases the isotropy subgroup is $\mathrm{SO}(D-2)$ and by spin one we mean it carries the vector representation of $\mathrm{SO}(D-2)$.

However, for the massless case the Wigner formulation of particle states involves introducing a gauge field and the procedure has a number of ad hoc steps associated with the gauge symmetry of this field. There does exist a much less well known, but equivalent formulation of the Wigner unitary irreducible representations, that is manifestly $\mathrm{SO}(1, D-1)$ covariant and, for the massless case, is also manifestly gauge invariant; indeed it involves field strengths and their derivatives and plays an important role in the formulation of nonlinear higher spin theories, see [22-26] and refs. therein. We will ask what possible gauge potentials are contained in this representation and in this way we will find an infinite possible choices of gauge potentials. In this section we take the opportunity to give a hopefully very readily understandable account of this formulation of the irreducible representations of $\operatorname{ISO}(1, D-1)$ using only knowledge that every physicists knows.

\subsection{The Wigner unitary irreducible representation of spin one}

As every theorist knows a spin one particle can be described by a rank two field strength $F_{a_{1} a_{2}}$ subject to the Bianchi identity

$$
\partial_{\left[a_{1}\right.} F_{\left.a_{2} a_{3}\right]}=0
$$


and the equation of motion

$$
\partial^{a} F_{a b}=0
$$

These imply that

$$
\partial^{a_{1}} \partial_{\left[a_{1}\right.} F_{\left.a_{2} a_{3}\right]}=0 " \text { and so } \partial^{b} \partial_{b} F_{a_{1} a_{2}}=0 .
$$

We will hence forth denote $F^{(0)} a_{1} a_{2}:=F_{a_{1} a_{2}}$ as this will be the first in a series of objects $F^{(n)}{ }_{a_{1} a_{2} \| b_{1} \ldots b_{n}}$ that we will define. We refer to $n$ as the level.

To show that these do indeed describe a spin one we can choose our Lorentz frame so that $k^{\mu}=\left(k^{+}, 0,0, \ldots, 0\right)$ in light-cone coordinates, whereupon equation (2.2) implies that $F_{+a}^{(0)}=0$ while equation (2.1) implies that $k_{[-} F^{(0)}{ }_{a b]}=0$. Consequently, the only non zero components of the field strength are $F^{(0)}{ }_{-i}, i=1, \ldots, D-2$ subject to equation (2.3) and these we recognise as the $D-2$ degrees of freedom of a "spin 1 ".

We are now going to formulate the above conditions in an alternative manner which will lead to the irreducible unitary representation of $\operatorname{ISO}(1, D-1)$ corresponding to spin one, but in such a way that it is manifestly $\mathrm{SO}(1, D-1)$ covariant and also gauge invariant. We first observe that the conditions of equation (2.1) and (2.2) on $F^{(0)} a_{1} a_{2}$ can be rewritten by defining

$$
F^{(1)}{ }_{a_{1} a_{2} \| b}:=\partial_{b} F^{(0)}{ }_{a_{1} a_{2}},
$$

whereupon they are equivalent to the conditions

$$
F_{\left[a_{1} a_{2} \| b\right]}^{(1)}=0=F^{(1)}{ }_{a_{1} b \|^{b}} .
$$

In the above and in what follows, we use conventions whereby double bars separate groups of indices that are subject to $\operatorname{GL}(D)$-irreducibility conditions. Thus we recognise the Bianchi identity of equations (2.1) as just being the requirement that the tensor $F^{(1)} a_{1} a_{2} \| b$ is $\mathrm{GL}(D)$ irreducible. This is the same as stating that $F^{(1)}{ }_{a_{1} a_{2} \| b}$ belongs to the $\mathrm{GL}(D)$ Young tableau

$$
\begin{array}{|l|l|}
\hline a_{1} & b \\
\hline a_{2} &
\end{array} .
$$

The second condition of equation (2.2) can be stated as that $F^{(1)} a_{1} a_{2} \| b$ is also a $\mathrm{SO}(1, D-1)$ irreducible tensor. A Young tableau can be of $\mathrm{GL}(D)$ or $\mathrm{SO}(1, D-1)$ type. The former encodes constraints that involve the antisymmetrisation, or symmetrisation, of certain groups of indices, such as in the first of the equation in (2.5), however, the latter tableau also encodes trace conditions, such as in the second equations in (2.5). As a result, $F^{(1)} a_{1} a_{2} \mid b$ belongs to the irreducible representation associated with the $\mathrm{SO}(1, D-1)$ Young tableau given above in (2.6). The conditions encoded in the Young tableau are just those required to give an irreducible representation of the relevant group. A discussion a Young tableaux can be found in $[29,30]$.

We now take another derivative and consider the quantity

$$
F^{(2)}{ }_{a_{1} a_{2} \| b_{1} b_{2}}:=\partial_{b_{2}} F^{(1)}{ }_{a_{1} a_{2} \| b_{1}},
$$

which satisfies the conditions

$$
F_{\left[a_{1} a_{2} \| b_{1}\right] b_{2}}^{(2)}=0=F^{(2)}{ }_{a_{1} b \|^{b} b_{2}}, \quad F^{(2)}{ }_{a_{1} a_{2} \|\left[b_{1} b_{2}\right]}=0=F^{(2)}{ }_{a_{1} a_{2} \| b}^{b} .
$$


The first two conditions are obvious from the definition of equation (2.7) and equation (2.5) while the last two conditions follow by substituting equation (2.4) into equation (2.7) and using equation (2.3). By considering $\left.\partial^{b_{1}} \partial_{\left[b_{1}\right.} F^{(1)} b_{2} b_{3} \| b_{4}\right]$, it follows from the constraints of equations (2.5) and (2.8) that

$$
\partial_{c} \partial^{c} F^{(1)}{ }_{a_{1} a_{2} \| b}=0=\partial_{c} \partial^{c} F^{(2)}{ }_{a_{1} a_{2} \| b_{1} b_{2}} .
$$

The constraints of equation (2.8) are equivalent to demanding that $F^{(2)} a_{1} a_{2} \| b_{1} b_{2}$ has the properties associated with the $\mathrm{SO}(1, D-1)$ Young tableau given by

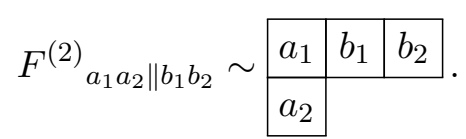

We now generalise the above to higher levels and define a sequence of objects up to level $n$ :

$$
\left\{F^{(p)}{ }_{a_{1} a_{2} \| b_{1} \ldots b_{p}}\right\}, \quad p=0,1, \ldots n,
$$

where we assume that

$$
F^{(p)}{ }_{\left[a_{1} a_{2} \| b_{1}\right] \ldots b_{p}}=0=F^{(p)}{ }_{a_{1} b \|^{b} b_{2} \ldots b_{p}}, F^{(p)}{ }_{a_{1} a_{2} \| b_{1} \ldots b_{p-2} c}{ }^{c}=0, \quad p=0,1, \ldots n,
$$

and

$$
F^{(p)}{ }_{a_{1} a_{2} \| b_{1} \ldots b_{p}}=F^{(p)}{ }_{a_{1} a_{2} \|\left(b_{1} \ldots b_{p}\right)}, \quad p=0,1, \ldots, n .
$$

Proceeding to the next level $n+1$ we define

$$
F^{(n+1)}{ }_{a_{1} a_{2} \| b_{1} \ldots b_{n+1}}:=\partial_{b_{n+1}} F_{a_{1} a_{2} \| b_{1} \ldots b_{n}}^{(n)} .
$$

It is now straightforward to show that $F^{(n+1)} a_{1} a_{2} \| b_{1} \ldots b_{n+1}$ obeys equations (2.12) and (2.13) but with $p=n+1$. Thus by induction we have an infinite set of objects which obey the constraints of equations (2.12) and (2.13) for all $p$.

To summarise, one has a description of "spin one" in $D$ dimensions in terms of an infinite the set of objects

$$
\mathcal{W}=\left\{\left.F^{(n)}{ }_{a_{1} a_{2} \| b_{1} \ldots b_{n}} \sim|l| l\right|_{a_{1}} b_{1} \ldots b_{n}, \quad n=0,1, \ldots\right\}
$$

which are related by equation (2.14) and which are subject to the constraints that are encoded in the $\mathrm{SO}(1, D-1)$ Young tableau.

The discussion above of all the higher level objects may seem at first sight as a bit redundant, but it has an important interpretation. The objects of equation (2.15) carry Wigner's unitary irreducible representation of $\operatorname{ISO}(1, D-1)$ which corresponds to "spin one". Indeed, there exists a map from Wigner's unitary irreducible representation of $\operatorname{ISO}(1, D-1)$ for "spin one" where all states are labelled by momentum and polarisation tensors, to $\mathcal{W}$, where the states are labelled by Lorentz tensors. The action of the Lorentz generators is as usual while the translations acts as

$$
P_{c}\left(F^{(n)}{ }_{a_{1} a_{2} \| b_{1} \ldots b_{n}}\right)=F^{(n+1)}{ }_{a_{1} a_{2} \| b_{1} \ldots b_{n} c} .
$$


The reader may verify that $\mathcal{W}$ does indeed carry a representation of $\operatorname{ISO}(1, D-1)$. We note that this representation is not irreducible, as it contains infinitely many ideals $\mathcal{W}_{n_{0}}$, namely the modules obtained by truncating the level $n$ to any minimum value $n_{0}$. As we shall see below, it is nevertheless possible to reconstruct $\mathcal{W}$ from any ideal $\mathcal{W}_{n_{0}}$ by integration with suitable boundary conditions imposed, conditions that we shall leave unspecified below for the sake of simplicity.

As we have mentioned, the advantage of using the above representation $\mathcal{W}$ and its generalisations to particles of other spin, is that it is manifestly Lorentz covariant and in the massless case also gauge invariant and so it does not require a particular representation in terms of a gauge potential and its associated gauge transformations. This will prove key in what follows. The representations $\mathcal{W}$, and its generalisations are equivalent to the formulation of these representations given by the Wigner method of induced representations $[17,18]$.

The discussion above was pedagogical but to some extent a simplified account using just ideas that are universally known. In fact, the procedure is best understood from a slightly different and more abstract viewpoint. We should start from the beginning with the fully indecomposable $\operatorname{ISO}(1, D-1)$ representation of equation $(2.15)$, the fields of which by definition are subject to the $\mathrm{SO}(1, D-1)$ conditions encoded in the Young tableaux, and related by the derivative condition of equation (2.14). As should be the case for this representation, these equations imply the on-shell dynamics. This should be apparent from the above, for example the constraints on $F^{(1)} a_{1} a_{2} \| b$ and the fact that $\partial_{b} F^{(1)} a_{1} a_{2}=F^{(1)} a_{1} a_{2} \| b$ implies the usual Bianchi and equation of motion for a spin one particle. The above Lorentz-covariant method of describing the particle states is an example of what is called the unfolded description of field theory dynamics and was initiated by M. Vasiliev, see $[22,23]$ and references therein. In particular, the unfolding of the spin-one field presented in this section was worked out in the second reference of $[22,23]$.

This is a formulation of the dynamics by a set of first order differential equations; in this case equations (2.14) for all $n$ together with the constraints just discussed. As we said, unfolded formulation plays the central role in nonlinear higher spin gravity theories.

The representation $\mathcal{W}$ of equation (2.15) contains the field $F^{(0)}{ }_{a_{1} a_{2}}$ and all its derivatives and one can think of this as the field and all its derivatives at a given space-time point. Using Taylor's theorem, we then know the fields at all space-time points as the coefficients in the expansion are the just mentioned quantities.

It is clear from the above construction that if we have the representation $\mathcal{W}$ up to level $n$ then we can, by acting with space-time derivatives, construct all the higher level elements in the representation; indeed this is what we did above. However, it is also possible to reconstruct $\mathcal{W}$ if we have all the elements at, and above, any given level $n$, which we denoted $\mathcal{W}_{n}$ above. The reconstruction is possible by using the Poincaré lemma. The fact that integration is required is to be expected, as $\mathcal{W}$ is a fully indecomposable representation. Let us consider $F^{(p)}{ }_{a_{1} a_{2} \mid b_{1} \ldots b_{p}}, \quad p \geq n$, which is subject to all the constraints dictated by its $\mathrm{SO}(1, D-1)$ Young tableau of equation (2.15). These, in particular, imply that

$$
\partial_{\left[b_{n+1} \mid\right.} F^{(n)}{ }_{\left.a_{1} a_{2} \| b_{1} \ldots \mid b_{n}\right]}=0 .
$$


As a result one can deduce, using the usual Poincaré lemma, that there exists an object $F^{(n-1)}{ }_{a_{1} a_{2} \| b_{1} \ldots b_{n-1}}$ such that

$$
F^{(n)}{ }_{a_{1} a_{2} \| b_{1} \ldots b_{n}}=\partial_{b_{n}} F^{(n-1)}{ }_{a_{1} a_{2} \| b_{1} \ldots b_{n-1}} .
$$

From the fact that $F^{(n)} a_{1} a_{2} \| b_{1} \ldots b_{n}$ satisfies the algebraic constraints associated with its $\mathrm{SO}(1, D-1)$ Young tableau, it follows that $F^{(n-1)}{ }_{a_{1} a_{2} \| b_{1} \ldots b_{n-1}}$ obeys the analogous constraints. Proceeding in this way we reconstruct the representation down to level zero.

It is instructive to find the degrees of freedom contained in the higher level elements of the representation $\mathcal{W}$. Let us consider $F_{a_{1} a_{2} \| b}^{(1)}$ which is subject to the constraints of equation (2.8) in conjunction with equation (2.7), but not its connection to level zero, that is, to $F_{a_{1} a_{2} \| b}^{(1)}$ of equation (2.4). These differential constraints imply that $F_{a_{1} a_{2} \| b}^{(1)}$ is divergenceless and curl-free on its two sets of indices, and as a result $F_{a_{1} a_{2} \| b}^{(1)}$ is harmonic. One goes to momentum space and takes $k^{\mu}=\left(k^{+}, 0,0, \ldots, 0\right)$, as before, and finds that the last three equations imply that the indices $a_{1}, a_{2}$ and $b$ cannot take the value + . The curlfree equations then imply that the only non-zero components are $F_{-i \|-}^{(1)}, \quad i=1, \ldots, D-2$. Hence we find it contains the required $D-2$ degrees of freedom. A similar analysis at level $n$ implies that the only non-zero components of $F^{(n)} a_{1} a_{2} \| b_{1} \ldots b_{n}$ are $F_{-i \|-\cdots-}^{(n)}$. We note that, in the chosen Lorentz frame, all the non-vanishing components are related by $F_{-i \|-\cdots-}^{(n)}=k_{-} \ldots k_{-} F_{-i}^{(0)}$ and reproduce all the Taylor coefficients of an on-shell Maxwell field at any given point, therefore allowing to reconstruct the field in the neighbourhood of that point.

\subsection{Dualities and Gauge potentials}

The representation $\mathcal{W}$ of $\operatorname{ISO}(1, D-1)$ describes the states of a spin one in a way that is manifestly gauge invariant, since it is constructed from field strengths and their derivatives. We now consider what gauge potentials are implied by this representation. We begin at the lowest level. Every theorist knows that the Bianchi identity of equation (2.1) can be solved in terms of a gauge potential $A_{a}^{(0)}$ as

$$
F^{(0)}{ }_{a_{1} a_{2}}=2 \partial_{\left[a_{1}\right.} A^{(0)}{ }_{\left.a_{2}\right]},
$$

with the usual gauge symmetry $\delta A_{a}=\partial_{a} \Lambda$.

However, we are free to choose which of the equations in the representation we would like to solve and we can equally well choose to solve equation (2.2) even though this is usually thought of as the equation of motion. To achieve this we define

$$
G_{a_{1} \ldots a_{D-2}}=\frac{1}{2} \epsilon_{a_{1} \ldots a_{D-2}}{ }^{b_{1} b_{2}} F_{b_{1} b_{2}},
$$

whereupon the equation (2.2) becomes

$$
\epsilon^{a_{1} a_{2} b_{1} \ldots b_{D-2}} \partial_{a_{2}} G_{b_{1} \ldots b_{D-2}}=0,
$$

with the solution

$$
G_{b_{1} \ldots b_{D-2}}=\partial_{\left[b_{1}\right.} A^{(0)}{ }_{\left.b_{2} \ldots b_{D-2}\right]}
$$


that is, in terms of a gauge field $A_{b_{1} \ldots b_{D-3}}^{(0)}$ with the gauge symmetry

$$
\delta_{\lambda} A_{b_{1} \ldots b_{D-3}}^{(0)}=\partial_{\left[b_{1}\right.} \lambda_{\left.b_{2} \ldots b_{D-3}\right]} .
$$

However as we can reconstruct $\mathcal{W}$ from $\mathcal{W}_{n}$ by integration, we choose to carry out a duality at level $n$ rather than those at level zero. Let us first consider level one and define

$$
G^{(1)}{ }_{c_{1} \ldots c_{D-1} \| a_{1} a_{2}}:=\epsilon_{c_{1} \ldots c_{D-1} b} F^{(1)} a_{1} a_{2} \|^{b} .
$$

It is straightforward to verify, using equation (2.2), that is, the trace constraint $F^{(1)} a b \|^{b}$ $=\partial^{b} F_{a b}=0$, that

$$
G^{(1)}{ }_{\left[c_{1} \ldots c_{D-1} \| a_{1}\right] a_{2}}=0,
$$

Thus, $G^{(1)} c_{1} \ldots c_{D-1} \| a_{1} a_{2}$ sits inside the irreducible representation of $\operatorname{GL}(D)$ that transforms according to the following $\operatorname{GL}(D)$ Young tableau

$$
\begin{gathered}
G^{(1)}{ }_{c_{1} \ldots c_{D-1} \| a_{1} a_{2}} \sim \begin{array}{c|c|}
\hline c_{1} & a_{1} \\
\hline c_{2} & a_{2} \\
\hline \vdots \\
c_{D-1}
\end{array} .
\end{gathered}
$$

However, it is easy to see that $G^{(1)}{ }_{c_{1} \ldots c_{D-2} b \|^{b}} a_{2} \neq 0$ and so the constraints on this object are not those of an $\mathrm{SO}(1, D-1)$ Young tableau, which are single traceless by definition. It does however satisfy a higher order trace condition, and using the first equation of (2.5), one finds that

$$
G^{(1)}{ }_{a_{1} a_{2} c_{1} \ldots c_{D-3} \|^{a_{1} a_{2}}}=0 .
$$

We can think of equation (2.27) as the equation of motion and equation (2.25) as the Bianchi identity for the particle when written in terms of $G^{(1)}{ }_{c_{1} \ldots c_{D-1}} \| a_{1} a_{2}$. Indeed we will show below that equation (2.27) follows from extremising an action. We note the usual interchange of equation of motion and Bianchi identity under a duality transformation.

We next consider what derivative constraints $G^{(1)}{ }_{c_{1} \ldots c_{D-1} \| a_{1} a_{2}}$ satisfies. Using equation (2.8) and the definition (2.24) we find that

$$
\partial_{\left[a_{1}\right.} G^{(1)}{ }_{\left.c_{1} \ldots c_{D-1} \| a_{2} a_{3}\right]}=0=\partial_{\left[c_{1}\right.} G_{\left.{ }_{2} \ldots c_{D}\right] \| a_{1} a_{2}}^{(1)},
$$

and

$$
\partial^{e} G^{(1)}{ }_{c_{1} \ldots c_{D-1} \| e a}=0=\partial^{d} G^{(1)}{ }_{d c_{2} \ldots c_{D-1} \| a_{1} a_{2}} .
$$

Equations (2.28) can be summarised by defining

$$
G_{c_{1} \ldots c_{D-1}\left\|a_{1} a_{2}\right\| b}^{(2)}:=\partial_{b} G^{(1)}{ }_{c_{1} \ldots c_{D-1} \| a_{2} a_{3}},
$$


and demanding that it belong to the $\mathrm{GL}(D)$ Young tableau

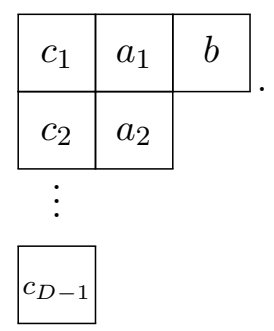

The tensor $G_{c_{1} \ldots c_{D-1}\left\|a_{1} a_{2}\right\| b}^{(2)}$ satisfies trace conditions inherited from equation (2.29) and an obvious second order trace condition inherited from (2.27). Because of the latter condition, these are not the single-trace conditions associated with an $\mathrm{SO}(1, D-1)$-irreducible Young tableau.

Before introducing potentials, we can proceed a but further as we did for $F_{a_{1} a_{2}}$ to construct an infinite dimensional representation based on $G_{c_{1} \ldots c_{D-1} \| a_{1} a_{2}}^{(1)}$ by defining

$$
G_{c_{1} \ldots c_{D-1}\left\|a_{1} a_{2}\right\| b_{1} \ldots b_{n+1}}^{(n+1)}:=\partial_{b_{n+1}} G^{(n)}{ }_{c_{1} \ldots c_{D-1}}\left\|a_{2} a_{3}\right\| b_{1} \ldots b_{n},
$$

and find the constraints that the new objects satisfies. However, we will not pursue this further in this work.

We now choose to regard equations (2.28) as Bianchi identities and so solve these instead of the Bianchi identity at level zero. Using the generalised Poincaré lemma spelled out in section 5 of [31], we find that

$$
G^{(1)}{ }_{c_{1} \ldots c_{D-1} \|}{ }^{a_{2} a_{3}}=\partial^{\left[a_{1}\right.} \partial_{\left[c_{1}\right.} A^{(1)}{ }_{\left.c_{2} \ldots c_{D-1}\right] \|}{ }^{\left.a_{2}\right]} .
$$

Thus we find a description in terms of a gauge field $A_{a_{1} \ldots a_{D-2} \| b}$ which satisfies the GL $(D)$ irreducibility condition

$$
A_{\left[a_{1} \ldots a_{D-2} \| b\right]}^{(1)}=0 .
$$

The expression for the field strength in terms of the gauge field can be written in the form of a Young tableau as follows

$$
\begin{array}{c|c|}
\hline \partial_{c_{1}} & \partial_{a_{1}} \\
\hline c_{2} & a_{2} \\
\hline \multicolumn{2}{|c}{} \\
\multicolumn{2}{|c}{c_{D-1}} \\
\end{array}
$$

The fields strength $G^{(1)}{ }_{c_{1} \ldots c_{D-1} \| a_{2} a_{3}}$ is invariant under the following gauge transformations featuring two independent GL $(D)$-irreducible gauge parameters $\lambda^{(1)}{ }_{a_{1} a_{2} \ldots a_{D-3} \| b}$ and $\lambda^{(2)}{ }_{a_{1} a_{2} \ldots a_{D-2}}$ :

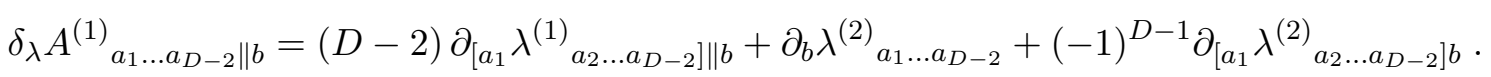


These two transformations can be represented by the tableaux
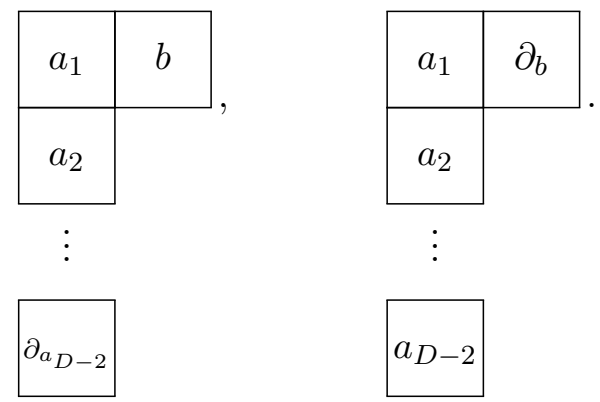

We now turn to the case of dualising the object in the irreducible representation of $\mathrm{SO}(1, D-1)$ given in equation $(2.15)$ at level $n$. To this end, we need a more streamlined index notation. We denote $A_{a[n]} \equiv A_{\left[a_{1} \ldots a_{n}\right]} \equiv A_{a_{1} \ldots a_{n}}$ and similarly for all blocks of antisymmetric indices. Similarly, for groups of symmetric indices, we use $S_{a(n)} \equiv S_{\left(a_{1} \ldots a_{n}\right)} \equiv$ $S_{a_{1} \ldots a_{n}}$, with strength-one (anti)symmetrisation convention. Using this notation we define

$$
G^{(n)}{ }_{c^{1}[D-1]\|\ldots\| c^{n}[D-1] \| a_{1} a_{2}}:=\epsilon_{c^{1}[D-1] e_{1}} \ldots \epsilon_{c^{n}[D-1] e_{n}} F_{a_{1} a_{2} \|}^{(n)}{ }^{e(n)} .
$$

Using equations (2.12) and (2.13) one can show that $G^{(n)}{ }_{c^{1}}[D-1]\|\ldots\| c^{n}[D-1] \| a_{1} a_{2}$ obeys the following over-antisymmetrisation constraints

$$
\begin{aligned}
& G_{{ }^{1}[D-1] \| \ldots . .}^{(n)}{ }_{D}^{1} c^{i}[D-2]\|\ldots\| c^{n}[D-1] \| a_{1} a_{2} \\
& i \in\{2, \ldots, n\}, \quad j \in\{1,2, \ldots, n\} .
\end{aligned}
$$

As a result, $G^{(n)}$ belongs to the $\mathrm{GL}(D)$ Young tableau

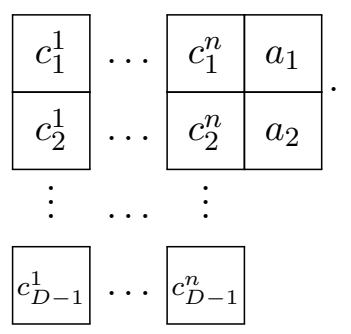

It is straightforward to show that although $G^{(n)}{ }_{c[D-1] \| \ldots . .}\|d[D-1]\| a_{1} a_{2}$ does not satisfy any single trace conditions it does satisfy a double and a $(D-1)$-trace condition which are given by

$$
G^{(n)}{ }_{c[D-1]\|\ldots . .\| d[D-3] a_{1} a_{2} \|}^{a_{1} a_{2}}=0=G^{(n)}{ }_{c[D-1]\|\ldots\|^{c[D-1]}}{ }^{\left[a_{1} a_{2}\right.} .
$$

The dynamics of the "spin one" when expressed in terms of the field strength $G^{(n)}{ }_{c[D-1]\|\ldots . . d[D-1]\| a_{1} a_{2}}$ is given by equations (2.39) and (2.41) which replace equations (2.1) and (2.2) of the usual formulation in terms of the field strength $F_{a_{1} a_{2}}^{(0)}$. We can think of equation (2.39) as generalised Bianchi identities at level $n-1$ and equations (2.41), which involve traces, as equations of motion. 
Using the equations (2.38) and equation (2.13), we find that the field strength $G^{(n)}{ }_{c[D-1]\|\ldots\| d[D-1] \| a_{1} a_{2}}$ also obeys the curl-free conditions

$$
\partial_{c} G^{(n)}{ }_{c[D-1]\|\ldots\| d[D-1] \| a_{1} a_{2}}=0=\partial_{a_{1}} G^{(n)}{ }_{c[D-1]\|\ldots\| d[D-1] \| a_{2} a_{3}},
$$

as well as the divergence-free conditions

$$
\partial^{e} G^{(n)}{ }_{e c[D-2]\|\ldots\|}\|d[D-1]\| a_{1} a_{2}=0=\partial^{e} G^{(n)}{ }_{c[D-1]\|\ldots\| d[D-1] \| e a} .
$$

We note that (2.42) will be the generalised Bianchi identities at the level $n$ while the equation of motion will not be (2.43) but instead the higher-trace constraints (2.39).

We find the gauge potential at level $n$ by applying the generalised Poincaré lemma [31] to equation (2.42). The result is

$$
\left.\left.G_{\left.c^{1}[D-1]\right]\|\ldots\| c^{n}[D-1] \|}^{(n)}{ }^{a_{1} a_{2}}=\partial^{\left[a_{1}\right.} \partial_{\left[c_{1}^{1}\right.} \ldots \partial_{\left[c_{1}^{n}\right.} A^{(n)} c^{1}[D-2]\right]\|\ldots\| c^{n}[D-2]\right]\|\|^{\left.a_{2}\right]},
$$

where the gauge potential $A^{(n)}{ }_{c[D-2]\|\ldots\| d[D-2] \| a}$ is an irreducible tensor of $\mathrm{GL}(D)$ and so obeys the constraints

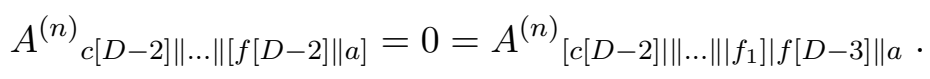

As a result $A_{c[D-2]\|\ldots\| f[D-2] \| a}^{(n)}$ belongs to the GL(D) Young tableau

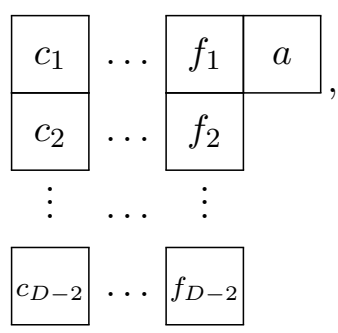

The potential has no trace constraint and has gauge symmetries involving two gauge parameters, $\Lambda_{[D-2, \ldots, D-2]}^{(n, 1)}$ and $\Lambda_{[D-2, \ldots, D-2, D-3,1]}^{(n, 2)}$.

We note that the field strength $G^{(n)}{ }_{c[D-1]]\|\ldots . .\| d[D-1] \| a_{1} a_{2}}$ involves $n+1$ space-time derivatives, instead of the more familiar two derivatives. The expression of the field strength $G^{(n)}{ }_{c[D-1]]\|\ldots . . f[D-1]\| a_{1} a_{2}}$ in terms of the gauge field $A^{(n)}{ }_{c[D-2]\|\ldots . . f[D-2]\| a}$ given in equation (2.44) can be expressed in Young tableau language as

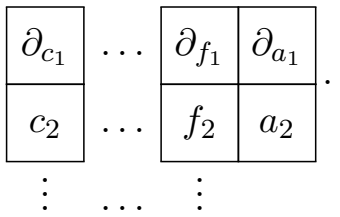

$$
\begin{aligned}
& \begin{array}{|l|l|l|}
\hline c_{D-2} & \ldots & f_{D-2} \\
\cline { 1 - 1 } c_{D-1} & \ldots & f_{D-1} \\
\hline
\end{array}
\end{aligned}
$$

The fields strength $G^{(n)}{ }_{c[D-1]]\|\ldots . .\| d[D-1] \| a_{1} a_{2}}$ when expressed in terms of its gauge potential in equation (2.44) automatically obeys the Bianchi identities of equations (2.39) 
and (2.42). However, it also obeys equation (2.41) which is the equation of motion for the gauge field, and we stress that it contains $n+1$ space-time derivatives. The presence of higher space-time derivatives is characteristic of the equations of motion of higher spin fields and indeed of any mixed symmetry fields, when formulated in terms of curvatures. For a recent discussion and references along those lines, see [32].

Thus we have found that there is an infinite number of ways of representing the particle states of "spin one" corresponding to the existence of an infinite number of different possible gauge potentials arising from the infinite number of ways of dualising the field strength and its descendants that occur in the Lorentz-covariant unfolded module $\mathcal{W}$. We repeat that the latter module carries the irreducible unitary representation of $\operatorname{ISO}(1, D-1)$ that describes the states of the "spin one".

We now turn to a key point of this paper, which is the duality relations between the first-order derivatives of the potentials. To obtain these, we will first obtain duality relation between the $(n+1)$-derivative field strengths when they are expressed in terms of their respective gauge potentials. We begin at level zero and in particular equation (2.20) which now relates the gauge field $A_{a}$ to the gauge field $A_{a[D-3]}$. This duality relation is of a familiar type in so much as it relates equations of motion to Bianchi identities. However, once we have substituted in the gauge potentials the Bianchi identities hold automatically and so the relations imply the equations of motion.

We now consider duality relation at level $n=1$, which was given in equation (2.24) and that can be written as

$$
\partial_{\left[a_{1}\right.} \partial_{\left[c_{1}\right.} A^{(1)}{ }_{\left.c[D-2]] \| a_{2}\right]}=\epsilon_{c[D-1] b} \partial^{b} \partial_{\left[a_{1}\right.} A^{(0)}{ }_{\left.a_{2}\right]} .
$$

We note that the Bianchi identities of equations of (2.1), or equivalently the first equation in (2.5), and equation (2.25) are automatically satisfied. However as the duality relation interchange Bianchi identities with equations of motion for the two fields we find that equation (2.48) automatically imposes the equations of motion of the two fields, namely, the second equation of (2.5) and equation (2.27), i.e.

$$
\partial^{b} F_{a b}=0
$$

and

$$
\partial^{a_{1}} \partial_{\left[a_{1}\right.} A^{(1)}{ }_{\left.a_{2} c[D-3]\right] \|}^{a_{2}}=0 .
$$

Another, more direct way of getting these two equations directly from (2.48) is to antisymmetrise all the $c$ indices together with $a_{1}$ of that equation, which gives (2.49), or to take its double trace, which gives of (2.50).

We now consider the duality relations at higher levels. We begin with the relation (2.38) but write it in the form

$$
\begin{aligned}
G_{c[D-1]\|\ldots\| b[D-1] \| a_{1} a_{2}}^{(n)} & =\epsilon_{b[D-1]}{ }^{f} G^{(n-1)}{ }_{c[D-1]\|\ldots\| a_{1} a_{2} \| f} \\
& =\epsilon_{b[D-1]}{ }^{f} \partial_{f} G^{(n-1)}{ }_{c[D-1]\|\ldots\| a_{1} a_{2}},
\end{aligned}
$$

which relates field strengths at adjacent levels. We now examine the effect of imposing the Bianchi identities and equations of motion on each of these field strengths without 
assuming that they are given in terms of the gauge fields. For indices that are not involved in the duality, that is do not occur on the epsilon symbol, the constraints on one side of the equation obviously hold on the other side. As a result we now consider the constraints that involve indices that occur in the duality. The Bianchi identities of $G^{(n)}{ }_{c[D-1]\|\ldots\| b[D-1] \| a_{1} a_{2}}$ of equation (2.39) imply the trace conditions for $G^{(n-1)}{ }_{c[D-1]\|\ldots\| a_{1} a_{2} \| f}$, namely

$$
G_{c[D-1]\|\ldots\|\left[b[D-1] \| a_{1}\right] a_{2}}^{(n)}=0 \quad \Longleftrightarrow \quad G^{(n-1)}{ }_{c[D-1]\|\ldots .\| a_{1} b \|^{b}=0}
$$

and

$$
G_{\left[c[D-1]\|\ldots\| b_{1}\right] b[D-2] \| a_{1} a_{2}}^{(n)}=0 \quad \Longleftrightarrow \quad G^{(n-1)}{ }_{e c[D-2]\|\ldots\| a_{1} a_{2} \|} \|^{e}=0 .
$$

Conversely the Bianchi identities of $G^{(n-1)}{ }_{c[D-1] \| \ldots . .} a_{1} a_{2} \| f$ imply the trace conditions of $G^{(n)}{ }_{c[D-1]\|\ldots\| b[D-1] \| a_{1} a_{2}}$ of equation (2.41), namely

$$
G_{\left[c[D-1]\left|\|\ldots\| a_{1} a_{2} \|\right| f\right]}^{(n-1)}=0 \quad \Longleftrightarrow \quad G^{(n)}{ }_{c[D-1]\|\ldots\|^{c[D-1]}}{ }_{\| a_{1} a_{2}}=0
$$

and

$$
G_{c[D-1]\|\ldots\|\left[a_{1} a_{2} \| f\right]}^{(n-1)}=0 \quad \Longleftrightarrow \quad G^{(n)}{ }_{c[D-1]\|\ldots .\| a_{1} a_{2} b[D-3]\|\|^{a_{1} a_{2}}=0 .}
$$

Substituting for the gauge field in the duality relation of equation (2.51) yields

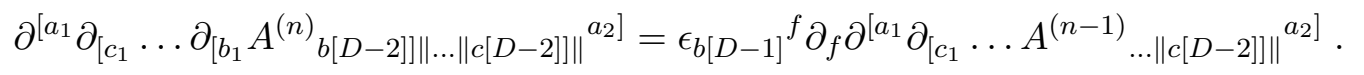

Once we have substituted the gauge fields in the field strengths, the Bianchi identities, which occur on the left hand-sides of equations (2.52)-(2.55), are automatically satisfied and as a result the trace conditions on the dual field strengths are now enforced. In particular, examining equation (2.54) and (2.55), we now find that their left-hand sides vanish automatically and so the gauge field $A_{[D-1]\|\ldots\| a}^{(n-1)}$ does not appear in this relation. Consequently, the right-hand side of these relations are enforced and we find that the field strength $G^{(n)}{ }_{c[D-1]\|\ldots . .\| b[D-1] \| a_{1} a_{2}}$ satisfy the trace conditions, which are the equations of motion for the gauge field $A_{c[D-2]\|\ldots\| b[D-1] \| a}^{(n)}$.

We note that the field strength is symmetric under the exchanges of its columns of $D-1$ indices and so the trace conditions hold on all these columns and not just for the the ones displayed above. Hence the duality condition of equation (2.56) implies the equation of motion for the "spin one" in the formulation with the level $n$ gauge field. Examining equations (2.52) and (2.53) we find a similar conclusion but now the gauge field $A_{c[D-2]\|\ldots b[D-1]\| a}^{(n)}$ is eliminated and we have the equation of motion for the gauge field $A_{c[D-2]\|\ldots b[D-2]\| a}^{(n-1)}$ field.

Equations (2.56) can be thought of as an infinite set of duality relations for $n=1,2, \ldots$, the first of which is given in equation (2.48). We note that they involve ever increasing numbers of space-time derivatives as $n$ increases. However, as we now show we can integrate these equations such that they only involve a single space-time derivative. At the lowest level we find, integrating equation (2.48), that

$$
\partial_{c} A_{c[D-2] \| a}^{(1)}=\epsilon_{c[D-1] b} \partial^{b} A_{a}^{(0)}{ }_{a}+\partial_{a} \Xi_{c[D-1]},
$$


where the last term is the general solution of the homogeneous equation. We can rewrite equation (2.57) as

$$
\partial_{c_{1}} A^{(1)}{ }_{c[D-2] \| a}=\epsilon_{c[D-1] b}\left\{\partial^{b} A^{(0)}{ }_{a}+\partial_{a} \Xi^{b}\right\}
$$

where $\Xi_{c[D-1]}=\epsilon_{c[D-1] b} \Xi^{b}$. However, since $\Xi^{b}$ is arbitrary, we can shift it as $\Xi^{b} \rightarrow \Xi^{b}-A^{b}$ whereupon our original equation becomes

$$
\partial_{c} A^{(1)}{ }_{c[D-2] \| a}=\epsilon_{c[D-1]^{b}} \partial_{[b} A^{(0)}{ }_{a]}+\partial_{a} \Xi_{c[D-1]},
$$

We observe that the equation is now invariant under the gauge transformations of the original gauge field $A^{(0)}{ }_{a}$. Antisymmetrising on $\left\{c_{1}, \ldots, c_{D-1}, a\right\}$, we find that $\partial_{c} \Xi_{c[D-1]}=$ 0 and so $\Xi_{c[D-1]}=\partial_{c} \Xi_{c[D-2]}$. Substituting this back in equation (2.57) it becomes

$$
\partial_{c_{1}} A^{(1)}{ }_{c[D-2] \| a}=\epsilon_{c[D-1]}{ }^{b} 2 \partial_{[b} A_{a]}^{(0)}+\partial_{a} \partial_{c} \Xi_{c[D-2]} .
$$

We recognise that the presence of the last term ensures the invariance of (2.60) under the gauge transformation of the second type in equation (2.37), which acts as a shift symmetry on $\Xi_{c[D-2]}$. The price for the integration is that the equation is now gauge-invariant only at the price of an extra field with a shift symmetry. To eliminate the extra field requires that we differentiate and antisymmetrise with the $a$ index, so recovering the original relation of equation (2.48).

Integrating at higher levels, in particular equation (2.56), we find that

$$
\begin{aligned}
\partial_{\left[b_{1}\right.} A_{b[D-2]]\left\|c^{1}[D-2]\right\| \ldots\left\|c^{n-1}[D-2]\right\| a}^{(n)}= & \epsilon_{b[D-1] f} \partial^{f} A^{(n-1)} c^{1}[D-2]\|\ldots\| c^{n-1}[D-2] \| a \\
& +Y\left(\partial_{a} \Sigma_{b[D-1] \mid c^{1}[D-2]\|\ldots\| c^{n-1}[D-2]}\right. \\
& \left.+\partial_{c^{n-1}} \Xi_{b[D-1] \mid c^{1}[D-2]\|\ldots\| c^{n-1}[D-3] \| a}\right), \\
n= & 0,1,2, \ldots,
\end{aligned}
$$

where $Y(\cdot)$ denotes the projection on the GL $(D)$ Young tableau with index structure $\left\{c^{1}[D-1]\|\ldots\| c^{n-1}[D-1] \| a\right\}$. Using arguments similar to those given below equation (2.57) one can bring the duality relation to a form that is invariant under certain of the gauge transformations of the field $A^{(n-1)} c_{1}[D-2]\|\ldots\| c_{n-1}[D-2] \| a$ and it then holds modulo the remaining gauge transformations of the two fields,

Rather than constructing the infinite set of duality relations beginning with the gauge field $A_{a}^{(0)}$ we can alternatively use the level zero gauge field $A_{b_{1} \ldots b_{D-3}}^{(0)}$ and repeat all the above steps. Including this step we find a formulation of the "spin one" field in terms of the following gauge fields

$$
\begin{array}{lllll}
A_{[1]}, & A_{[D-3]}, & A_{[D-2,1]}, & A_{[D-2, D-3]}, & A_{[D-2, D-2,1]}, \\
A_{[D-2, D-2, D-3]}, & \ldots, & A_{[D-2, \ldots, D-2,1]}, & A_{[D-2, \ldots, D-2, D-3]}, & \ldots
\end{array}
$$

where the numbers shown as subscripts between square brackets indicate the number of indices in each block, that is the length of columns in the corresponding Young tableau.

Thus, in summary we have shown that the spin one can be described by an infinite set of duality equations which are first order in space-time derivatives but only hold modulo 
certain gauge transformations, One might suspect that these duality relations are invariant under an infinite duality symmetry, modulo the gauge transformations. Indeed one might suppose that this can be formulated as a non-linear realisation of an algebra with generators that carry the same indices that are those carried by the gauge fields but raised.

\subsection{Action principle for the dual potential $A_{c[D-2] \| a}^{(1)}$}

In this section, we follow the lines sketched in [16] and give the action describing the dynamics of a Maxwell field in terms of the dual potential $A_{c[D-2] \| a}^{(1)}$ introduced above and sometimes denoted $A_{[D-2,1]}$, for the sake of brevity. The way we recover the dynamics (2.27) is interestingly subtle. As explained in the context of the Fierz-Pauli theory in [16], the various dual actions involve more and more off-shell fields and are therefore less and less economical. The special case of spin- 1 is simpler but allows us to see in a very explicit way the mechanism whereby the extra off-shell fields disappear from the dynamics on shell.

We start, as it should, with the Maxwell action, and integrate by part:

$$
S[A]=-\frac{1}{2} \int d^{D} x \partial_{a} A_{b}\left(\partial^{a} A^{b}-\partial^{b} A^{a}\right)=-\frac{1}{2} \int d^{D} x\left(\partial_{a} A_{b} \partial^{a} A^{b}-\partial_{a} A^{a} \partial_{b} A^{b}\right),
$$

dropping the boundary term. Introducing the following parent action

$$
S[Y, P]=\int d^{D} x\left(P_{a \mid}{ }^{b} \partial_{c} Y^{c a \mid}{ }_{b}-\frac{1}{2} P^{a}{ }_{\mid b} P^{b}{ }_{\mid a}+\frac{1}{2} P^{a}{ }_{\mid a} P^{b}{ }_{\mid b}\right)
$$

that features two fields, $Y^{c a \mid}{ }_{b}=-Y^{a c \mid}{ }_{b}$ and $P_{a \mid}{ }^{b}$, we reproduce the original action (2.63) upon extremising $S[Y, P]$ with respect to $Y$ :

$$
\partial_{[c} P_{a]\left.\right|^{b}}=0 \quad \Leftrightarrow \quad P_{a \mid}^{b}=\partial_{a} A^{b},
$$

and plugging back into (2.64). On the other hand, $P_{a \mid}^{b}$ is an auxiliary field, so that extremising the action with respect to it enables one to express it in terms of the $Y$ field:

$$
P_{b \mid}^{a}=\partial_{c} Y^{c a \mid}-\frac{1}{(D-1)} \delta_{b}^{a} \partial_{c} Y^{c d \mid} .
$$

Plugging that expression for $P$ inside the parent action (2.64) yields the action

$$
S\left[Y_{b}^{c a \mid}\right]=\int d^{D} x\left(\frac{1}{2} \partial_{c} Y^{c a \mid}{ }_{b} \partial^{d} Y_{d a \mid}{ }^{b}-\frac{1}{2(D-1)} \partial_{c} Y^{c a \mid}{ }_{a} \partial^{b} Y_{b d \mid}{ }^{d}\right) .
$$

In order to analyse the gauge invariances of the action, it is sufficient to use only a decomposition of the various fields under $\operatorname{GL}(D)$ and not under $O(1, D-1)$. Thus, we decompose

$$
Y_{c}^{\left.a b\right|_{c}}=X^{a b \mid}{ }_{c}+\delta_{c}^{[a} Z^{b]}, \quad X^{a b \mid}{ }_{a} \equiv 0 .
$$

The invariance of the Maxwell action under the gauge transformation $\delta A_{a}=\partial_{a} \lambda$ is inherited by the new action (2.67), whereby the field $Z$ transforms as $\delta Z_{a}=\partial_{a} \lambda$, with $X^{a b}{ }_{c}$ staying unchanged, i.e. the action (2.67) can be shown to be invariant under

$$
\delta_{\lambda} Y^{a b \mid}{ }_{c}=\delta_{c}^{[a} \partial^{b]} \lambda .
$$


On the other hand, from the fact that the field $Y^{a b \mid}{ }_{c}$ enters the action (2.67) only through its divergence $\partial_{c} Y^{c a \mid}{ }_{b}$, the action is manifestly invariant under the following gauge transformation

$$
\delta_{\Upsilon} Y^{\left.a b\right|_{c}}=\partial_{d} \Upsilon^{d a b \mid}{ }_{c}, \quad \Upsilon^{\left.d a b\right|_{c}} \equiv \Upsilon^{\left.[d a b]\right|_{c}} .
$$

Using the invariant antisymmetric symbol of $\operatorname{SL}(D)$ to dualise the first two indices of $Y^{a b \mid}{ }_{c}$, the decomposition (2.68) is tantamount to the following GL(D)-irreducible decomposition

$$
\begin{aligned}
& \widetilde{Y}_{a[D-2] \mid c}=T_{a[D-2] \| c}+\widetilde{Z}_{a[D-2] c}, \quad T_{a[D-2] \| a} \equiv 0, \quad \widetilde{Z}_{a[D-2] c} \equiv \widetilde{Z}_{[a[D-2] c]}, \\
& \widetilde{Y}_{a[D-2] \| c}:=\frac{1}{2} \epsilon_{a[D]} Y^{a[2] \mid}, \quad \quad T_{a[D-2] \| c}:=\frac{1}{2} \epsilon_{a[D]} X^{a[2] \mid}, \quad \widetilde{Z}_{a[D-2] c}:=\frac{1}{2} \epsilon_{c b a[D-2]} Z^{b},
\end{aligned}
$$

while the gauge parameter $\Upsilon$ is similarly dualised into

$$
\widetilde{\Upsilon}_{a[D-3] \mid c}=\lambda_{a[D-2] \| c}^{(1)}+\lambda_{a[D-2] c}^{(2)}, \quad \lambda_{a[D-3] \| a}^{(1)} \equiv 0, \quad \lambda_{a[D-3] c}^{(2)} \equiv \lambda_{[a[D-3] c]}^{(2)} .
$$

At this stage, without losing any of the tensorial fields involved, we set $D=4$ for the sake of clarity and to further explain the gauge structure of the new action in terms of the GL(4)-irreducible dual fields $T_{[2,1]}$ and $Z_{[1]}$. The gauge transformations leaving the action (2.67) invariant, with $D=4$ and keeping the vector field $Z_{a}$ instead of its Hodge dual $\widetilde{Z}_{a[3]}$, now read

$$
\begin{aligned}
\delta T_{a b \| c} & =2 \partial_{[a} \lambda^{(1)}{ }_{b] c}-2 \partial_{[a} \lambda^{(2)}{ }_{b] c}+2 \partial_{c} \lambda^{(2)}{ }_{a b}, \\
\delta Z_{a} & =\partial_{a} \lambda+\partial^{b} \widetilde{\lambda}^{(2)}{ }_{a b}, \quad \widetilde{\lambda}_{a b}^{(2)}=\frac{1}{2} \epsilon_{a b c d} \lambda^{(2) c d},
\end{aligned}
$$

where $\lambda^{(1)}{ }_{a b}=\lambda^{(1)}{ }_{(a b)}$ and $\lambda^{(2)}{ }_{a b}=\lambda^{(2)}{ }_{[a b]}$.

In terms of the fields $X^{a b \mid}{ }_{c}$ and $Z_{a}$ that we keep for the moment, the equations of motion derived from (2.67) are

$$
0=G_{a c \mid}^{b}:=\frac{1}{2}\left(\partial^{b} F_{a c}(Z)+2 \partial^{d} \partial_{[c} X_{a]\left.d\right|^{b}}^{b}\right), \quad F_{a c}(Z):=2 \partial_{[a} Z_{c]} .
$$

When the field $X$ is expressed in terms of its dual $T$, in four dimensions, we have the field equations

$$
\partial_{b} F_{a c}(Z)+\frac{1}{12}\left[\epsilon_{a d u v} \partial_{c} F^{d u v \|_{b}}-\epsilon_{c d u v} \partial_{a} F^{d u v \|_{b}}\right]=0, \quad F_{a b c \| d}:=3 \partial_{[a} T_{b c] \| d},
$$

where we note that the curvature $F_{a b c \| d}$ of $T$ is invariant under the $\lambda^{(1)}$ gauge symmetry. Dualising on the indices $a c$ gives

$$
2 \partial_{d} \widetilde{F}^{a b}(Z)-\partial_{c} F_{d}^{a b c \|_{d}}=0, \quad \text { where } \quad \widetilde{F}^{a b}(Z):=\frac{1}{2} \epsilon^{a b c d} F_{c d}(Z) .
$$

Antisymmetrising the left-hand side of the equations of motion (2.75) in its free indices, one finds

$$
\partial_{a} F^{a b c \|_{c}}=0 \text {. }
$$


In this equation, only the GL(4)-irreducible field $T_{[2,1]}$ appears and all the symmetries in (2.73) are preserved. The above field equation is nothing but the equation

$$
G_{a_{1} a_{2} c \|}^{(1)} a_{1} a_{2}=0,
$$

presented in (2.27), in the case where $D=4$. We note that, using the Hodge decomposition whereby a differential $p$-form can be written as the sum of three terms, one d-exact, one $* d *$-exact and the last one harmonic:

$$
\omega_{[p]}=\mathrm{d} p_{[p-1]}+* \mathrm{~d} * q_{[p+1]}+r_{[p]}, \quad\{* \mathrm{~d} *, \mathrm{~d}\} r_{[p]}=0,
$$

the field $Z_{a}$ can be set to zero using the $\lambda$ and $\lambda^{(2)}$ gauge parameters, while its harmonic piece can be obtained by integrating equation (2.75), thereby expressing it in terms of the physical components of $T$. In the gauge where the closed and co-closed parts of $Z_{a}$ vanish, one cannot use any $\lambda^{(2)}$ gauge parameters anymore and the remaining action and field equations are only invariant under the $\lambda^{(1)}$ gauge symmetry.

\section{The three form in eleven dimensions}

The eleven dimensional supergravity theory as originally formulated contains the graviton and the three form as its bosonic sector [33]. How to formulate the eleven dimensional action with a six form was discussed in reference [34, 35]. The $E_{11}$ non-linear realisation in eleven dimensions includes the usual fields for the graviton and three form as well as the six form and a field which is the dual of the graviton, but in addition it contains an infinite number of fields with blocks of height nine added, see equation (1.1). Among these fields are the $h_{[9,9, \ldots, 9,8,1]}$. In this section we will repeat the considerations of the sections two, but for the three form. We will show how the alternative dual descriptions of the degrees of freedom usually encoded in the three form arise naturally within the unfolded formulation. We find the equations of motion of the theory when described by any of these dual gauge fields and we will find an infinite set of duality relations that are first order in space-time derivatives and encode the dynamics. As we will discuss in the Conclusions, these relations should be contained in the non-linear realisation based on $E_{11}$.

\subsection{The unfolded representation of the three-form}

In what follows we will construct the unfolded representation of the three-form, that is both $\mathrm{SO}(1,10)$ and gauge invariant. We recall that this representation is indecomposable, but can be mapped via harmonic expansion to Wigner's irreducible unitary representation of $\operatorname{ISO}(1,10)$ for the three form. It can be found following the unfolding procedure given in $[22,23]$, see also $[24,26]$ and references therein. Unlike in the previous section where we presented the unfolded formulation of Maxwell's theory using standard tensor calculus, in this section we give a more formal and compact account of the unfolded representation using differential form calculus and stress its conceptual basis.

Wigner's unitary irreducible representation of the Poincaré group $\operatorname{ISO}(1,10)$ corresponding to the free, dynamical three-form in eleven dimensions can be mapped to an 
unfolded module consisting of an infinite set $\mathcal{T}$ of $\mathrm{SO}(1,10)$-irreducible tensors

$$
\mathcal{T}=\left\{F_{a[4]}, F_{a[4] \| b}, F_{a[4] \| b(2)}, F_{a[4] \| b(3)}, \ldots\right\},
$$

where the notation $F_{a[4] \| b(n)}$ indicates a tensor that is separately antisymmetric in its four indices $\left\{a_{1}, a_{2}, a_{3}, a_{4}\right\}$ and totally symmetric in its $n$ indices $\left\{b_{1}, b_{2}, \ldots, b_{n}\right\}$. The $\mathrm{SO}(1,10)$-irreducibility of the tensors $\left\{F_{a[4] \| b(n)}, n=0,1, \ldots\right\}$ means that, besides being Young-projected, the tensors are traceless, viz.

$$
F_{a[4] \| a b(n-1)} \equiv 0, \quad \eta^{a_{1} b_{1}} F_{a[4] \| b(n)} \equiv 0,
$$

where we recall our convention that indices at the same position (covariant or contravariant) and with the same Latin label are implicitly symmetrised, or antisymmetrised, according to the context. We note that the difference between an GL(11) and an $\mathrm{SO}(1,10)$-irreducible tensor is given by the tracelessness property, here the second identity of (3.2).

In terms of Young tableau, the tensor $F_{a[4] \| b(n)}$ is represented by

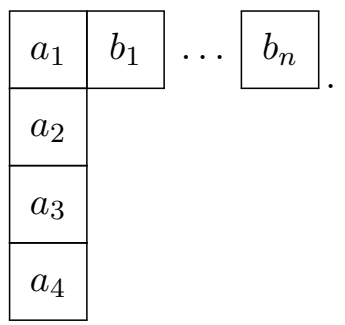

The action of the Poincaré group on the infinite set of tensors in (3.1) is given by

$$
P_{b} F_{a[4]}=F_{a[4] \| b}, \quad P_{b_{2}} F_{a[4] \| b_{1}}=F_{a[4] \| b_{1} b_{2}}, \quad P_{b_{3}} F_{a[4] \| b_{1} b_{2}}=F_{a[4] \| b_{1} b_{2} b_{3}}, \ldots
$$

while the Lorentz generators $M_{a b}$ act diagonally in $\mathcal{T}$ by the usual action. Up to this stage, although we have talked of tensors, we have used no notion of spacetime.

Introducing a spacetime, the action of the translation generators of the Poincaré group on the representation can be explicitly realised by taking them to be differentiation with respect to the space-time coordinates, that is, $P_{a}=\partial_{a}$, whereupon equations (3.4) take the form

$$
\begin{aligned}
\partial_{b} F_{a[4]} & =F_{a[4] \| b}, \\
\partial_{b_{2}} F_{a[4] \| b_{1}} & =F_{a[4] \| b_{1} b_{2}}, \\
\partial_{b_{3}} F_{a[4] \| b_{1} b_{2}} & =F_{a[4] \| b_{1} b_{2} b_{3}},
\end{aligned}
$$

The infinite set of differential equations (3.5)-(3.7) can be compactly written upon introducing Grassmann odd (resp. even) vector oscillators $\theta^{a}$ (resp. $u^{a}$ ) and forming the master field

$$
F(x ; \theta, u)=\sum_{n=0}^{\infty} \frac{1}{4 ! n !} F_{a[4] \| b(n)}(x) \theta^{a_{1}} \ldots \theta^{a_{4}} u^{b_{1}} \ldots u^{b_{n}}
$$


It is also advantageous to write everything in terms of differential forms, by using the total exterior derivative $\mathrm{d}=d x^{\mu} \partial_{\mu}$, taking the $F_{a[4] \| b(n)}$ to be zero forms and introducing the one-form

$$
h^{a}:=d x^{\mu} \delta_{\mu}^{a}
$$

for Minkowski spacetime in Cartesian coordinates. In this setting, the infinite set of differential equations (3.5)-(3.7) given above can be written in the form

$$
\left[\mathrm{d}-i h^{a} \rho_{\mathcal{T}}\left(P_{a}\right)\right] F(x ; \theta, u)=0,
$$

where the translation generators are now represented on the master field as follows:

$$
\rho_{\mathcal{T}}\left(P_{a}\right)=(-i) \frac{\partial}{\partial u^{a}} .
$$

Explicitly, equations (3.62)-(3.7) now read

$$
\begin{aligned}
\mathrm{d} F_{a[4]} & =h^{c} F_{a[4] \| c}, \\
\mathrm{~d} F_{a[4] \| b} & =h^{c} F_{a[4] \| b c}, \\
\mathrm{~d} F_{a[4] \| b(2)} & =h^{c} F_{a[4] \| b(2) c} .
\end{aligned}
$$

Taking into account the GL(11) irreducibility conditions, given in equation (3.2), of the tensor on the right-hand side of equation (3.5), one derives the relation

$$
\partial_{a} F_{a[4]}=0
$$

which is locally solved, as usual, by $F_{[4]}=\mathrm{d} A_{[3]}$, introducing a three-form potential and its four-form field strength

$$
A_{[3]}=\frac{1}{6} h^{a_{1}} \wedge h^{a_{2}} \wedge h^{a_{3}} A_{a[3]}, \quad F_{a[4]}:=\frac{1}{24} h^{a_{1}} \wedge \ldots \wedge h^{a_{4}} F_{a[4]} .
$$

We are using the notation that a number in square brackets without being accompanied by a letter denotes the degree of the form that the field belongs to, that is, $A_{[3]}$ is a form of degree three. The zero-form tensor $F_{a[4]}$ are thus the components of the four-form field strength $F_{[4]}=\mathrm{d} A_{[3]}$. As usual the gauge field $A_{[3]}$ is defined up to the exterior derivative of a two-form potential, namely

$$
A_{[3]} \sim A_{[3]}+\mathrm{d} \Lambda_{[2]} .
$$

On the other hand, recalling that $F_{a[4] \| b}$ obeys trace constraints as given in equation (3.2), one derives the equation

$$
\partial^{a} F_{a[4]}=0,
$$

which together with equation (3.16), is the field equation of a dynamical three-form. We also not that the other equations (3.13), (3.14) etc. can be solved one after the others. They express the tensors $F_{a[4] \| b(n)}$ as the higher gradients of the tensors $F_{a[4] \| b(m)}$ for $m<n$ and so in terms of the on-shell dynamical three-form $A_{[3]}$ :

$$
F_{a[4] \| b(n)}=4 \partial_{b_{1}} \partial_{b_{2}} \ldots \partial_{b_{n}} \partial_{\left[a_{1}\right.} A_{\left.a_{2} a_{3} a_{4}\right]} .
$$


We note that the $\mathrm{SO}(1,10)$ properties of $F_{a[4] \| b(n)}$ are ensured by the equations of motion of the three form and the fact that partial derivatives commute.

To summarise, the irreducible unitary representation of equation (3.1) contains components that are individually subject to $\mathrm{SO}(1, D-1)$ irreducibility conditions and once we take the space-time translations to be realised by space-time differentiation these conditions imply the well known equation of motion for a three form. This is a purely algebraic way of encoding the field equations and Bianchi identities of a dynamical three-form, a characteristic of unfolded dynamics.

The underlying algebraic structure, captured by (3.12)-(3.14) together with $d A_{[3]}=$ $\frac{1}{24} h^{a_{1}} \wedge \ldots \wedge h^{a_{4}} F_{a[4]}$ and $\mathrm{d} h^{a}=0$, is known as a free differential algebra and makes sense on a base manifold of arbitrary dimension. Its initial data is given by the gauge functions for $A_{[3]}$ and the vielbeins $h^{a}$ together with the infinite set of constants provided by the zero-forms at a given point $p_{0}$ of the manifold. In particular, in eleven dimensions, the infinite set of zero-forms in $\mathcal{T}$ at a point $p_{0}$ with Cartesian coordinates $x_{0}^{\mu}$, together with the differential equations (3.10), give the necessary data that enables one to reconstruct an on-shell, dynamical three-form around that point $p_{0}$ using the Taylor expansion

$$
A_{a[3]}(x)=A_{a[3]}\left(x_{0}\right)+\sum_{n=1}^{\infty} \frac{1}{n !}\left(x-x_{0}\right)^{b_{1}} \ldots\left(x-x_{0}\right)^{b_{n}} F_{a[3] b \| b(n-1)}\left(x_{0}\right) .
$$

We would like to make some comments on gauge fixing. In the light-cone coordinates $x^{\mu}=\left(x^{-}, x^{+}, x^{i}\right)$ we can choose the Lorentz frame in which the momentum is $k_{\mu}=$ $\left(k_{-}, k_{+}=0, k_{i}=0\right)$. Then at the point $p_{0}$, the components $A_{-j k}$ and $A_{-+j}$ can be set to zero by fixing the gauge in equation (3.17) using the gauge parameters $\lambda_{i j}$ and $\lambda_{+i}$. Furthermore, the components $A_{+i j}$ are gauge-invariant and zero on-shell as the field equation is given by $k_{-} A_{+i j}=0$. As a result one finds that the three-form potential has all its components vanishing except for the purely transverse ones, for which

$$
A_{i j k}\left(x_{0}\right)=\frac{1}{k_{-}} F_{-i j k}\left(x_{0}\right) .
$$

Consequently, all the derivatives of the three-form, when evaluated in momentum space and in the chosen Lorentz frame, are therefore given by all the powers of $k_{-}$times the Fourier transform of $A_{i j k}(x)$ and they transform in the following representation

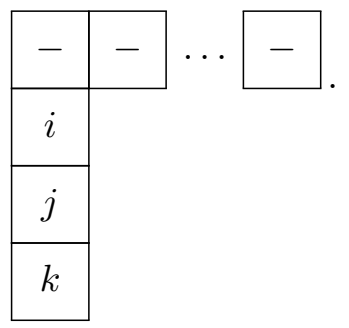

These coincide with all the non-vanishing on-shell derivatives of the field strength. This discussion follows the general arguments given in references [19-21] (for related discussions, see [32]) and it is the equivalent, for the three-form, of the Petrov decomposition of a metric in Riemannian geometry. 
We next note how the gauge-for-gauge transformations, $\delta \lambda_{[2]}=\mathrm{d} C_{[1]}$, act. In our chosen Lorentz frame the only gauge transformations that have a non-trivial gauge-forgauge transformation are $\lambda_{-i}$ and $\lambda_{-+}$. These are also the only gauge parameters which we did not use so far. They are subject to transformations that involve the components $C_{i}$ and $C_{+}$and these can be used to set these gauge parameters to zero, that is set $\lambda_{-i}=0=$ $\lambda_{-+}$. We note that the component $C_{-}$can be set to zero by the gauge-for-gauge-for-gauge parameter.

Another, alternative and Lorentz-covariant way of analysing the physical content of the equations consists in Taylor expanding the gauge (and higher reducibility) parameters, the three-form components as well as the field strength, all evaluated on-shell, and comparing all the coefficients of the various powers of $\left(x-x_{0}\right)$ at the point $p_{0}$. One sees that the constants $A_{a b c}\left(x_{0}\right)$ can be set to zero by the constants $\partial_{[a} \lambda_{b c]}\left(x_{0}\right)$ (the latter not being constrained by the reducibility transformations). Similarly, at first order in the derivatives of the three-form, the constants $\partial_{(a} A_{b) c d}\left(x_{0}\right)$ can be set to zero by the constants $\partial_{a} \partial_{[b} \lambda_{c d]}+$ $\partial_{b} \partial_{[a} \lambda_{c d]}$ whereas the constants $\partial_{[a} A_{b c d]}\left(x_{0}\right)$ are identified (up to a constant, irrelevant factor) with the constants $F_{a b c d}\left(x_{0}\right)$, etc. The outcome of this procedure is that all the derivatives $\partial_{c_{n}} \ldots \partial_{c_{1}} A_{a[3]}\left(x_{0}\right)$ of the three-form at the point $p_{0}$ are set equal to the on-shell derivatives $\partial_{\left(c_{n}\right.} \ldots \partial_{c_{2}} F_{\left.c_{1}\right) a[3]}\left(x_{0}\right)$, thereby explaining (3.20). This way of counting physical degrees of freedom on-shell is the one adopted in unfolded dynamics [22, 23].

It is well known that rather than describe the degrees of freedom by a three form one can use a 6 -form potential and we now explain this from the unfolded viewpoint. We begin with the relation

$$
F^{a[7]}:=\frac{1}{4 !} \epsilon^{a[7] b[4]} F_{b[4]},
$$

and transfer the properties of the unfolded dynamics of the three form given in equations (3.5)-(3.7) to corresponding equations for the six form. The first unfolded equation (3.5) transforms in the $[4,1]$-irrep of $\mathrm{SO}(1,10)$ and the resulting divergenceless property of $F_{a[4] \| b}$ implies that $F^{a[7]}$ is d-closed:

$$
0=\partial^{a} F_{a c[3]} \quad \Leftrightarrow \quad \partial^{a} F^{a[7]}=0,
$$

while the GL(11)-irreducibility of $F_{a[4] \| b}$, that is the Bianchi identity of $F_{a[4]}$, implies that $F^{a[7]}$ is divergenceless:

$$
\partial_{a} F_{a[4]} \equiv 0, \quad \Leftrightarrow \quad \partial_{b} F^{b a[6]}=0 .
$$

By the usual Poincaré lemma, equation (3.24) implies that $F^{a[7]}$ can locally be written as

$$
F^{a[7]}=7 \partial^{a} A^{a[6]} .
$$

Thus we find the usual exchange the equations of motion with the Bianchi identities in equations (3.24) and (3.25).

We now define $F_{a[7] \| b}$ by

$$
F_{a[7] \| b}:=\partial_{b} F_{a[7]} .
$$

By virtue of equations (3.24) and (3.25), $F_{a[7] \| b}$ is an irreducible $\mathrm{SO}(1,10)$ tensor as it is GL(11)-irreducible $\left(F_{a[7] \| a}=0\right)$ and traceless $\left(F_{a[6] b \|^{b}}=0\right)$. Completing the unfolding of 
the dual linearised 6-form yields the following tower of tensors

$$
\widetilde{\mathcal{T}}=\left\{F_{a[7] \| b(n)}, \quad n=0,1, \ldots,\right\} .
$$

The action of the Poincaré generators $P_{c}$ on the tensors in $\tilde{\mathcal{T}}$ is given by

$$
P_{c} F_{a[7] \| b(n)}=F_{a[7] \| c b(n)}=\partial_{c} F_{a[7] \| b(n)} .
$$

It follows from (3.23) and the above conventions for the action of the Poincaré translations that the tensors in $\widetilde{\mathcal{T}}$ of equation (3.28) and those in $\mathcal{T}$ of equation (3.1) are related by

$$
F_{a[7] \| b(n)}=\frac{1}{4 !} \epsilon^{a[7] c[4]} F_{c[4] \| b(n)}, \quad n=1,2, \ldots
$$

The tensors in $\widetilde{\mathcal{T}}$ are traceless as result of the relation

$$
F^{a[6] c \|_{c b(n-1)}}=\frac{1}{4 !} \epsilon^{a[6] c d[4]} F_{d[4] \| c b(n-1)}=0,
$$

and are GL(11)-irreducible as a consequence of

$$
\epsilon^{a[8] d[3]} F_{a[7] \| a b(n-1)}=\frac{1}{4 !} \epsilon^{a[8] d[3]} \epsilon_{a[7] c[4]} F^{c[4] \|}{ }_{a b(b-1)}=7 ! F^{d[3] a \|}{ }_{a b(n-1)}=0 .
$$

Hence the tensors $\widetilde{\mathcal{T}}$ of equation $(3.28)$ belong to the $\mathrm{SO}(1,10)$ Young tableau

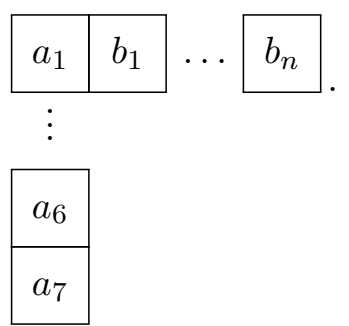

We can collect the tensors $\tilde{\mathcal{T}}$ into a single object

$$
F(x ; \theta, u)=\sum_{n=0}^{\infty} \frac{1}{7 ! n !} F_{a[7] \| b(n)}(x) \theta^{a_{1}} \ldots \theta^{a_{7}} u^{b_{1}} \ldots u^{b_{n}},
$$

for which equation (3.29) takes the form

$$
\left[\mathrm{d}-i h^{a} \rho_{\widetilde{\mathcal{T}}}\left(P_{a}\right)\right] F(x ; \theta, u)=0, \quad \text { where } \quad \rho_{\widetilde{\mathcal{T}}}\left(P_{a}\right)=(-i) \frac{\partial}{\partial u^{a}}=\rho_{\mathcal{T}}\left(P_{a}\right) .
$$

Although action principles are usually part of the definition of an unfolded system, it is nevertheless instructive to consider a parent action from which one can find both the action for the three gauge form and that for the six form gauge field:

$$
S\left[A_{[3]}, F_{[7]}\right]=\int\left(\mathrm{d} A_{[3]} \wedge F_{[7]}-\frac{1}{8} F_{[7]} \wedge * F_{[7]}\right),
$$

where $F_{[7]}$ and $A_{[3]}$ are independent fields. Extremising it with respect to $A_{[3]}$ gives $d F_{[7]}=$ 0 and so $F_{[7]}=\mathrm{d} A_{[6]}$; substituting this back in $S\left[A_{[3]}, F_{[7]}\right]$, gives the standard action 
$S\left[A_{[6]}\right] \propto \int \mathrm{d} A_{[6]} \wedge * \mathrm{~d} A_{[6]}$. The equation of motion for $F_{7}$ gives $F_{7} \propto * d A_{3}$ and substituting back we find the standard action for the three form.

Alternatively, one can start from the Palatini formulation for the 3-form,

$$
S\left[A_{[3]}, F^{a[4]}\right]=\int \frac{1}{7 !} \epsilon^{b[4] c[7]} h_{c_{1}} \wedge \ldots \wedge h_{c_{7}}\left(\mathrm{~d} A_{[3]}+\frac{1}{8} h_{c_{1}} h_{c_{2}} h_{c_{3}} h_{c_{4}} F^{c[4]}\right) F_{b[4]},
$$

where $F^{a[4]}$ is a zero-form and is an independent field and we recall that $h_{c}$ is defined in equation (3.9). Defining

$$
F_{[7]}:=\frac{1}{7 !} \epsilon^{b[4] c[7]} F_{b[4]} h_{c_{1}} \wedge \ldots \wedge h_{c_{7}},
$$

the Palatini action (3.37) becomes identical to the action (3.36). The latter action will be used in section 3.3 where we shall generalise the action principle given above for Maxwell theory to the case of the three form in eleven dimension and in the frame-like formulation.

\subsection{Further dualisation of the three form}

It is well-known that rather than express the dynamics of the bosonic non-gravitational degrees of freedom of eleven dimensional supergravity by a three-form gauge field one can instead use a six-form gauge field $A_{[6]}$, whose curvature $F_{[7]}$, at the linearised level, is just the Hodge dual of $F_{[4]}$. As explained in the introduction, the non-linear realisation of the Kac-Moody algebra $E_{11}$ leads not only to the usual fields of eleven dimensional supergravity as well as a six form and dual graviton field, but also to the infinite set of fields of equation (1.1) which were proposed to be equivalent ways of describing the dynamics [7]. In this section we will show how the next field on the duality chain of equation (1.1), the gauge field $A_{[9,3]}$, arises and we give its linearised dynamics. The duality relation involving the fields in the gravity sector was sketched in reference [16] and some indications that one might be able to do this for any massless particle were discuss in [36].

As we explained for Maxwell theory in the previous section, one can dualise any of the curvature tensors that occur in the unfolded formulation. Hence, instead of dualising the first tensor in the set $\mathcal{T}$ in (3.1), one may dualise the second tensor $F_{[4,1]}$ on its second column:

$$
G^{b[10]}{ }_{\| a[4]}=\epsilon^{b[10] c} F_{a[4] \| c},
$$

or equivalently

$$
F_{a[4] \| b}=-\frac{1}{10 !} \epsilon_{b c[10]} G^{c[10]}{ }_{\| a[4]} .
$$

We can now find what the constraints on $F_{a[4] \| b}$ imply for $G_{b[10] \| a[4]}$. Taking the trace of (3.40) and using the second equation in (3.2) we find that indeed,

$$
G_{b[10] \| b a[3]}=0
$$

while using the first equation in (3.2) and acting with $\epsilon^{a[4] b d[6]}$ on equation (3.40) we find the quartic trace constraint

$$
G^{b[6] a[4]}{ }_{\| a[4]} \equiv\left(\operatorname{Tr}_{12}\right)^{4} G_{[10,4]}=0 .
$$


The presence of a higher order trace condition is unusual when compared to the standard formulation of particle dynamics including Fronsdal's higher-spin dynamics, but is nevertheless known in the context of duality, see section 6 of [31] and in particular equation (6.19) therein. Equation (3.41) implies that the tensor $G_{a[10] \| b[4]}$ is an irreducible GL(11) tensor of type $[10,4]$. Of course it cannot be traceless, as requiring this would imply its vanishing, as is well-known in the representation theory of the orthogonal group, see e.g. the theorem of section 10-6 in [29], which is equivalent to the special case $m=0$ of Proposition 3 in [31] or Lemma 1 in the more recent paper [37]. We note that, as usual, the Bianchi identities and field equations get swopped under the dualisation.

We would now like to look at the differential constraints on $G_{b[10] \| a[4]}$ that arise from the differential constraints on $F_{a[4] \| c}$ of equations (3.5)-(3.7). The first of these equations implies that $\partial_{a} F_{a[4] \| b}=0$ which using equation (3.40) in turn implies that

$$
\partial_{a} G^{b[10]}{ }_{\| a[4]}=0 .
$$

As we did for the Maxwell case we can continue taking more space-time derivatives of the the field strength $G_{b[10] \| a[4]}$ to find an infinite set of tensors $\left\{G_{a[10]\|b[4]\| b(n)}, n=0,1, \ldots\right\}$. Using similar arguments we can transfer the properties of $F_{a[4] \| b(n)}$ to those new tensors to find that $\left\{G_{a[10] \mid b[4] \| b(n)}, n=0,1, \ldots\right\}$ are GL(11)-irreducible and obey the trace constraints

$$
\left(\operatorname{Tr}_{12}\right)^{4} G_{[10,4,1, \ldots, 1]}=0, \quad \operatorname{Tr}_{1 \mathrm{i}} G_{[10,4,1, \ldots, 1]}=0=\operatorname{Tr}_{2 \mathrm{i}} G_{[10,4,1, \ldots, 1]}, \quad i \in\{3, \ldots, n\} .
$$

The notation $\left(\operatorname{Tr}_{i j}\right)^{n}$ used here means that one takes $n$ traces on the columns $i$ and $j$.

Equation (3.43), combined with the GL(11) irreducibility of $G^{b[10]}{ }_{\| a[4]}$ implies, using the generalised Poincaré lemma [31], That it can locally be written as

$$
G^{b[10]}{ }_{\| a[4]}=\partial_{a} \partial^{b} A^{b[9]}{ }_{\| a[3]},
$$

where the GL(11)-irreducible tensor gauge field $A_{b[9] \| a[3]}$ is defined up to the gauge transformation

$$
\delta A_{a[9] \| b[3]}=9 \partial_{a} \Lambda_{a[8] \| b[3]}^{(1)}+3\left(\partial_{b} \Lambda_{a[9] \| b[2]}^{(2)}+\frac{9}{7} \partial_{a} \Lambda_{a[8] b \| b[2]}^{(2)}\right),
$$

with the two gauge parameters being GL(11)-irreducible with type $\Lambda^{(1)}{ }_{[8,3]}$ and $\Lambda^{(2)}{ }_{[9,2]}$. We note that there are no algebraic trace constraints on $A_{[9,3]}$, nor on its gauge parameters.

Remembering the expression $F_{a[4] \| b}=\partial_{b} \partial_{a} A_{a[3]}$, the definition (3.39) of $G_{a[10] \| b[4]}$ and the relation (3.45) give us the following duality relation:

$$
\partial^{a} A^{a[9] \|_{b[3]}}=\epsilon^{a[10] c} \partial_{c} A_{b[3]}+\partial_{b} \Xi^{\left.a[10]\right|_{b[2]}} .
$$

which is the analog of (2.57). We first note that $\Xi^{\left.a[10]\right|_{b[2]}}$ decomposes into

$$
\Xi^{a[10]{ }_{b[2]}}=\Xi^{(1) a[10]} \| b[2]+\epsilon^{a[10]} \Xi^{(2)}{ }_{b}
$$

and that a gauge transformation $A_{b[3]} \rightarrow A_{b[3]}+\partial_{b} \lambda_{b[2]}$ with $\lambda_{b[2]}=-x_{b} \Xi^{(2)}{ }_{b}$ enables one to eliminate the $\Xi^{(2)}$ component of $\Lambda$. Having done that, the equation (3.47) is now 
understood with a field $\Xi_{a[10] \| b[2]}$ obeying $\Xi_{a[10] \| a b}=0$. We can now reformulate this equation in the same manner as we did for equation (2.57). By shifting the arbitrary field $\Xi^{a[10] \|_{b[2]}}$ in an appropriate way, we can recast the equation in the form

$$
\partial^{a} A^{a[9] \|_{b[3]}}=\epsilon^{a[10] b} 4 \partial_{b} A_{b[3]]}+\partial_{b} \Xi_{b[2]}^{a[10] \|_{b}} .
$$

Multiplying by $\epsilon^{a[10] e}$ and tracing on $b_{1}$ and $e$, we find that $\partial^{a} \Xi^{a[10] \|_{b[2]}}=0$ implying that $\Xi^{a[10] \|_{b[2]}}=\partial^{a} \Xi^{a[9] \|_{b[2]}}$. Using this result equation (3.47) now becomes

$$
\partial^{a} A^{a[9] \|_{b[3]}}=\epsilon^{a[10] c} 4 \partial_{[c} A_{b[3]]}+\partial_{b} \partial^{a} \Xi^{a[9] \|_{b[2]}} .
$$

We recognise the last term as a gauge transformation of the field $A^{a[9] \|_{b[3]}}$. Alternatively, the above equation can be made fully gauge invariant by giving a shift symmetry to the field $\Xi$ under $\Lambda^{(2)}[9,2]$.

We now give an action principle for the $A_{b[9] \| a[3]}$ potential that correctly describes the degrees of freedom of a massless three-form. The procedure was proposed in [16], which itself was inspired from [6,9]. We start with the three-form and follow the analog of the procedure for the Maxwell field spelled out in section 2.3. To this end, we take the usual action $S\left[A_{[3]}\right]$ for a three form and integrate by parts, ignoring boundary terms:

$$
-\frac{1}{4 !} \int d^{D} x \partial_{a} A_{a[3]} \partial^{a} A^{a[3]}=-\frac{1}{3 !} \int d^{D} x\left(\partial_{b} A_{a[3]} \partial^{b} A^{a[3]}+3 \partial^{b} A_{b a[2]} \partial_{c} A^{c a[2]}\right) .
$$

We then introduce the following parent action, that features two independent fields, $P_{b \mid a[3]}$ and $Y^{b[2] \mid a[3] \text { : }}$

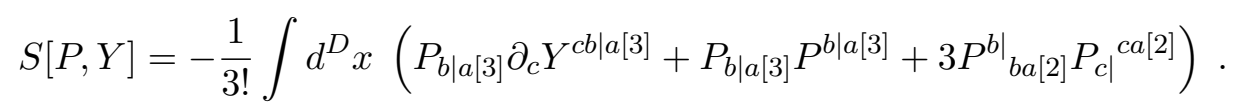

Varying the action $S[P, Y]$ with respect to the field $Y^{a[2] \mid b[3]}$ gives the equation $\partial_{b_{1}} P_{b_{2} \mid a[3]}=$ 0 which implies that $P_{b \mid a[3]}=\partial_{b} A_{a[3]}$. Substituted inside the action, we reproduce the action (3.51). On the other hand, as the field $P_{b \mid a[3]}$ is auxiliary one can express it in terms of $Y$ via its equation of motion, namely

$$
2 P^{b \mid a[3]}=-\partial_{c} Y^{c b \mid a[3]}-\frac{3}{D-1} \eta^{b a} \partial_{c} Y_{d}^{c d \mid}{ }^{a[2]},
$$

and substitute for it into the parent action, thereby yielding a daughter action $S\left[Y^{b[2] \mid a[3]}\right]$ expressed solely in terms of the field $Y$ :

$$
\begin{aligned}
S\left[Y^{c b \mid a[3]}\right]=\frac{1}{4 !} \int d^{D} x( & P_{b \mid a[3]} \partial_{c} Y^{c b \mid a[3]}+\partial_{c} Y^{c b \mid a[3]} \partial^{e} Y_{e b \mid a[3]} \\
& \left.-\frac{3\left(5 D^{2}-11 D+7\right)}{(D-1)^{2}} \partial_{c} Y_{b a[2]}^{c b \mid} \partial^{e} Y_{e d \mid} d a[2]\right) .
\end{aligned}
$$

Setting $D=11$, one can then dualise $Y^{a[2]}{ }_{\mid b[3]}$ on its first two indices, and decompose

$$
\widetilde{Y}_{a[9] \mid b[3]}=\frac{1}{2} \epsilon_{a[9] c[2]} Y_{\mid b[3]}^{c[2]}=A_{a[9] \| b[3]}+B_{a[9] b \| b[2]}+\epsilon_{a[9] b[2]} C_{b},
$$


so as to produce the GL(11)-irreducible field $A_{a[9] \| b[3]}$ satisfying $A_{a[9] \| a b[2]} \equiv 0$, as well as $B_{a[10] \| b[2]}$ (satisfying $B_{a[10] \| a b} \equiv 0$ ) and $C_{a}$ which are analogs of the field $\widetilde{Z}$ in equation (2.71). Because the field $Y^{c b \mid a[3]}$ enters the action only through its divergence $\partial_{c} Y^{c b \mid a[3]}$, the action is invariant under the following gauge transformations

$$
\delta Y^{b[2] \mid a[3]}=\partial_{c} \Upsilon^{c[3] \mid a[3]},
$$

where the gauge parameter $\Upsilon$ is antisymmetric in its two groups of indices. Upon dualising the parameter $\Upsilon$, one gets the following GL(11)-irreducible gauge parameters

$$
\frac{1}{3 !} \epsilon_{c[3]} \Upsilon^{c[3] \mid a[3]} \longrightarrow\left\{\Lambda_{a[8] \| b[3]}^{(1)}, \Lambda_{a[9] \| b[2]}^{(2)}, \Lambda_{a[10] \| b}^{(3)}, \Lambda^{(4)}\right\}
$$

The field $A_{a[9] \| b[3]}$ will then transform as in (3.46), while the gauge transformation of the field $B_{a[10] \| b[2]}$ will involve the gradient of the parameters $\Lambda^{(2)}$ and $\Lambda^{(3)}$. Finally, the vector field $C_{a}$ will transform with the gradient of $\Lambda^{(4)}$.

We note that the action also possesses the gauge symmetry involving the two-form gauge parameter $\lambda_{a[2]}$ inherited from the original three-form $A_{a[3]}$. This will be discussed in the next section 3.3, where we use the frame-like formalism that brings in a better insight into the gauge structure. On-shell, the gauge field $A_{a[9] \| b[3]}$ will obey the equation (3.42) discussed above.

\subsection{Unfolded description containing the $A_{[9,3]}$ form}

In this section we wish to construct the unfolded formulation of the dynamics for the $A_{[9,3]}$ form, that is a set of first order differential equations that contain the gauge field $A_{a[9]|| b[3]}$ and that assumes the form of a free differential algebra. This will contain the manifestly Lorentz covariant and gauge-invariant infinite-dimensional representation of $\operatorname{ISO}(1, D-1)$ constructed from the field strength, $G_{a[10] \| b[4]}$, and all of its higher on-shell derivatives. It also contains the gauge field $A_{a[9] \| b[3]}$ through an appropriate frame-like, or Cartan-like, connection. In the next subsection 3.4, we will build an action principle for the $A_{a[9] \| b[3]}$ potential, but this time facilitated by the use of the frame-like description that we first derive on-shell in the present subsection.

We first introduce, following [24], the connection-like objects

$$
\left\{e_{[9]}^{a[3]}, \omega_{[3]}^{a[10]}\right\} \text {. }
$$

The indices in square brackets without a label, i.e. [3] and [9], denote the form degree of the objects, for example $e_{[9]}{ }^{a[3]}$ is a nine form that carries three antisymmetrised tangent indices and so can be written in more usual notation as $\frac{1}{9 !} h^{b_{1}} \wedge \ldots \wedge h^{b_{9}} e_{b_{1} \ldots b_{9}} a_{1} a_{2} a_{3}$. The field $\omega_{[3]}^{a[10]}$ is a three form that carries ten antisymmetrised tangent indices. It is important to note that the objects of equation (3.58) are not subject to any $\mathrm{GL}(D)$ irreducibility conditions. By analogy with the vielbein formulation of general relativity, we may think of $e_{[9]}^{a[3]}$ as a generalised vielbein and $\omega_{[3]}^{a[10]}$ as a generalised spin-connection. As the field $e_{[9]}{ }^{a[3]}$ is not $\mathrm{GL}(D)$ irreducible, only one of its irreducible components can be identified with the gauge potential $A_{b[9] \| a[3]}$ that we considered in section 3.2; the precise identification will be discussed below. 
The differential forms of equation (3.58) are required to satisfy the differential equations

$$
\begin{aligned}
\mathrm{d} e_{[9]}^{a[3]}+h^{b_{1}} \wedge \ldots \wedge h^{b_{7}} \wedge \omega_{[3]}^{a[3]}{ }_{b[7]}=0, \\
\mathrm{~d} \omega_{[3]}{ }^{a[10]}+h^{c_{1}} \wedge \ldots \wedge h^{c_{4}} G^{a[10] \|_{c[4]}}=0,
\end{aligned}
$$

where by assumption $G^{a[10] \|_{c[4]}}$ is the zero-form that appeared in (3.39). It obeys the GL(D) irreducibility conditions and the higher-trace constraints of equations (3.41), (3.42). As discussed (3.43), this zero-form is the first member of an infinite set of zero-forms obeying the following first-order differential constraints:

$$
\begin{aligned}
\mathrm{d} G^{a[10] \| b[4]}+h_{c} G^{a[10]\|b[4]\| c} & =0, \\
\mathrm{~d} G^{a[10]\|b[4]\| c}+h_{c} G^{a[10]\|b[4]\| c(2)} & =0, \\
\vdots & \\
\mathrm{d} G^{a[10]\|b[4]\| c(n)}+h_{c} G^{a[10]\|b[4]\| c(n+1)} & =0, \quad n=2,3, \ldots .
\end{aligned}
$$

The equations (3.59)-(3.63) together with $d h^{a}=0$ form a free differential algebra and provides the unfolded description of the dual $A_{[9,3]}$ metric-like gauge field.

The gauge transformations of the system (3.59)-(3.60) are

$$
\begin{aligned}
\delta_{\epsilon} e_{[9]}^{a[3]} & =\mathrm{d} \epsilon_{[8]}^{a[3]}+h_{a_{1}} \wedge \ldots \wedge h_{a_{7}} \wedge \epsilon_{[2]}^{a[10]}=0, \\
\delta_{\epsilon} \omega_{[3]}^{a[10]} & =\mathrm{d} \epsilon_{[2]}^{a[10]} .
\end{aligned}
$$

The algebraic, Stückelberg-like, gauge transformations on $e_{[9]}^{a[3]}$, that is those contained in $\epsilon_{[2]}^{a[10]}$, can be used to gauge away certain components of $e_{[9]}^{a[3]}$. The GL(11)-irreducible decompositions of $e_{[9]^{a[3]}}$ and $\epsilon_{[2]}^{a[10]}$ are respectively given by

$$
[9] \otimes[3] \cong[11,1] \oplus[10,2] \oplus[9,3],
$$

and

$$
[10] \otimes[2] \cong[11,1] \oplus[10,2]
$$

Therefore, after using all the algebraic gauge symmetries, the remaining components in $e_{[9]}^{a[3]}$ are contained in the GL(11)-irreducible gauge field $A_{a[9] \| b[3]}$. Thus we make the connection with the equations of motion of section 3.2 which involved the GL $(D)$-irreducible gauge field $A_{a[9] \| b[3]}$. The connection $\omega_{[3]}^{a[10]}$ possesses two GL(11)-irreducible pieces: $[10,3] \oplus[11,2]$. However, it is determined from the "zero-torsion" equation (3.59) by the first derivatives of the components of $\epsilon_{[9]}^{a[3]}$ that can be reduced (or gauge-fixed) to its $A_{a[9] \| b[3]}$ part. As a result we find that only the $[10,3]$ irreducible component of $\omega_{[3]}^{a[10]}$ remains that we denote by $\tilde{\omega}_{a[10] \| b[3]}$.

In summary, so far, equations (3.61)-(3.63) constrain a tower of manifestly Lorentzcovariant and gauge-invariant zero forms $\left\{G^{(n)}, n=0,1, \ldots\right\}$ such that these can be expanded in terms of the unitary and irreducible massless representation of $\operatorname{ISO}(1, D-1)$ that describes the degrees of freedom propagated by the original three form gauge field. 
This is simply a consequence of the fact that the field strength $G^{a[10] \| b[4]}$ is by assumption expressed in terms of the field strength $F_{a[4]}$ via (3.39), so the representation appearing in (3.61)-(3.63) is equivalent to the representation built on the field strength $F_{a[4]}$ contained in equation (3.1). Equations (3.59) and (3.60) glue the zero-form tower to the gauge field $e_{[9]}^{a[3]}$ thanks to the introduction of the generalised spin connection $\omega_{[3]}^{a[10]}$ so as to write the full system as a free differential algebra. We will show later in this section how to reproduce an equivalent dynamics from an action principle involving the fields in (3.58) with some additional zero-forms.

In order to make contact with the gauge parameters of the metric-like $A_{[9,3]}$ gauge fields, we note that, as is typical for $p$-form systems such as a nine-form and a 3 -form, the gauge transformations admit reducibility transformations. The complete family of gauge-for-gauge $p$-form parameters, which are not $\mathrm{GL}(D)$ irreducible, is given by:

$$
\left\{\epsilon_{[9-i]}^{a[3]}\right\}, \quad i=1,2, \ldots, 9
$$

and

$$
\left\{\epsilon_{[3-j]}^{a[10]}\right\}, \quad j=1,2,3
$$

with transformation rules

$$
\begin{aligned}
\delta \epsilon_{[8]}^{a[3]}=\mathrm{d} \epsilon_{[7]}^{a[3]}+h_{a_{1}} \wedge \ldots \wedge h_{a_{7}} \wedge \epsilon_{[1]}^{a[10]}, & \delta_{\epsilon}^{a[3]}=\mathrm{d} \epsilon_{[6]}^{a[3]}+h_{a_{1}}^{a[3]} \wedge \wedge h_{a_{7}} \wedge \epsilon_{[0]}^{a[10]}, \\
\delta \epsilon_{[2]}^{a[10]}=\mathrm{d} \epsilon_{[1]}^{a[10]}, \quad \delta \epsilon_{[1]}^{a[10]}=\mathrm{d} \epsilon_{[0]}^{a[10]}, & \delta \epsilon_{[0]}^{a[10]}=0,
\end{aligned}
$$

and

$$
\delta \epsilon_{[6]}^{a[3]}=\mathrm{d} \epsilon_{[5]}^{a[3]}, \quad \delta \epsilon_{[5]}^{a[3]}=\mathrm{d} \epsilon_{[4]}^{a[3]}, \ldots, \quad \delta \epsilon_{[1]}^{a[3]}=\mathrm{d} \epsilon_{[0]}^{a[3]}, \quad \delta \epsilon_{[0]}^{a[3]}=0 .
$$

The gauge-for-gauge parameter $\epsilon_{[1]}^{a[10]}$ can be used to gauge away parts of the parameter $\epsilon_{[8]}^{a[3]}$. Both are GL(11) reducible and can be decomposed into the GL(11) representations as follows

$$
[10] \otimes[1] \cong[11] \oplus[10,1], \quad[8] \otimes[3] \cong[11] \oplus[10,1] \oplus[9,2] \oplus[8,3]
$$

As this decomposition makes clear we can gauge away two components leaving the gauge parameter $\epsilon_{[8]}^{a[3]}$ to contain only the GL(11)-irreducible representation $[9,2] \oplus[8,3]$. Making the appropriate GL(11) projection on equation (3.64), we find that the gauge transformation of the $A_{[9,3]}$ potential takes the form:

$$
\delta_{\epsilon} A_{a[9] \|}{ }^{b 3]}=9 \partial_{a} \epsilon_{a[8] \|}{ }^{b 3]}+3\left(\partial^{b} \epsilon_{a[9] \|}{ }^{b[2]}+\frac{9}{7} \partial^{b} \epsilon_{a[8]}{ }^{b \| b} a\right),
$$

thereby making contact with (3.46).

When equations (3.59)-(3.60) are reduced to the remaining GL(11)-irreducible components $A_{a[9] \| b[3]}$ and $\tilde{\omega}_{a[10] \| b[3]}$ of $e_{[9]}^{a[3]}$ and $\omega_{[3]}^{a[10]}$, they become

$$
\begin{aligned}
\tilde{\omega}_{a[10] \| b[3]} & =\partial_{a} A_{a[9] \| b[3]}, \\
\partial^{b} \tilde{\omega}_{a[10] \|} b[3] & =G_{a[10] \|} b[4]
\end{aligned}
$$


The expression of the field strength in terms of the gauge field is given by

$$
\partial^{b} \partial_{a} A_{a[9] \|}^{b[3]}=G_{a[10] \|}{ }^{b[4]},
$$

which agrees with equation (3.45). It is easy to see that it is invariant under the gauge transformations (3.74).

\subsection{First-order frame-like action for the $A_{[9,3]}$ field}

We now follow the general procedure explained in [36], whose discussion for the spin-2 case was already given in [38]. The action, just like the one given at the end of section 3.3 , is a parent action in the sense that it contains both the three form and the $A_{[9,3]}$ gauge field. The difference between this action and the one presented in section 3.3 is that we will now use the frame-like vantage point developed above for the gauge field $A_{a[9] \| b[3]}$. We start from the action principle for the three-form, written in the Palatini formulation presented at the end of section 3.1 and that we repeat here for convenience:

$$
S\left[A_{[3]}, F^{a[4]}\right]=\int_{\mathcal{M}_{11}} \frac{1}{7 !} \epsilon^{b[4] c[7]} h_{c_{1}} \wedge \ldots \wedge h_{c_{7}}\left(\mathrm{~d} A_{[3]}+\frac{1}{8} h_{c_{1}} h_{c_{2}} h_{c_{3}} h_{c_{4}} F^{c[4]}\right) F_{b[4]},
$$

where $A_{[3]}$ and $F_{a[4]}$ are independent fields. We next introduce the parent action

$$
\begin{aligned}
S^{P}\left[A_{[3]}, F_{a[4]}, t_{[1]}^{a[3]}, e_{[9]}^{a[3]}\right]=\int_{\mathcal{M}_{11}}\left[\frac{1}{7 !} \epsilon^{b[4] c[7]} h_{c_{1}} \wedge \ldots \wedge h_{c_{7}}\right. \\
\left.\wedge\left(\mathrm{d} A_{[3]}+\frac{1}{8} h_{c_{1}} h_{c_{2}} h_{c_{3}} h_{c_{4}} F^{c[4]}+t_{[1]}^{c[3]} h_{c_{1}} h_{c_{2}} h_{c_{3}}\right) F_{b[4]}+t_{[1] a[3]} \mathrm{d} e_{[9]}^{a[3]}\right],
\end{aligned}
$$

that contains the additional independent fields $t_{[1] a[3]}$ and $e_{[9]}^{a[3]}$. The field equations derived from the parent action are given by

$$
\begin{aligned}
\mathrm{d} A_{[3]}+\frac{1}{4} h_{c_{1}} h_{c_{2}} h_{c_{3}} h_{c_{4}} F^{c[4]}+t_{[1]}^{c[3]} h_{c_{1}} h_{c_{2}} h_{c_{3}} & =0, \\
\mathrm{~d} *\left(h^{a_{1}} \ldots h^{a_{4}} F_{a[4]}\right) & =0, \\
\mathrm{~d} t_{[1]}[3] & =0, \\
\mathrm{~d} e_{[9]}^{a[3]}+\frac{1}{7 !} h^{a_{1}} h^{a_{2}} h^{a_{3}} F_{b[4]} \epsilon^{b[4] c[7]} h_{c_{1}} \wedge \ldots \wedge h_{c_{7}} & =0 .
\end{aligned}
$$

The gauge symmetries of the action are

$$
\begin{aligned}
\delta A_{[3]} & =\mathrm{d} \lambda_{[2]}-h_{c_{1}} h_{c_{2}} h_{c_{3}} \psi_{[0]}^{c[3]}, \\
\delta F_{[0]}^{a[4]} & =0 \\
\delta t_{[1]}^{a[3]} & =\mathrm{d} \psi_{[0]}^{a[3]} \\
\delta e_{[9]^{a[3]}} & =\mathrm{d} \xi_{[8]}{ }^{a[3]} .
\end{aligned}
$$

The equation (3.82) results from extremising with respect to $e_{[9]}^{a[3]}$. It implies that $t_{[1]}^{a[3]}=$ $\mathrm{d} C^{a[3]}$ and substituting this into the action we can absorb $C^{a[3]}$ into $A_{[3]}$ and the action becomes that of equation (3.78). 
In order to descend on a different child action, we note that by using the Stückelberglike gauge symmetry of $A_{[3]}$ with gauge parameter $\psi_{[0]}^{a[3]}$, one can completely gauge $A_{[3]}$ away, so that it disappears from the action (3.79). One can still perform differential gauge transformations with the two-form gauge parameter $\lambda_{[2]}$, but in order to stay in the gauge where $A_{[3]}$ is zero, one has to compensate it with a residual transformation with parameter $\bar{\psi}^{a[3]}=\partial^{\left[a_{1}\right.} \lambda^{\left.a_{2} a_{3}\right]}$.

We are thus left with a child action containing the fields, $e_{[9]}^{a[3]}, F_{a[4]}$ and $t_{[1]}^{a[3]}$, or better, containing only $e_{[9]}^{a[3]}$ and $t_{[1]}^{a[3]}$ as $F_{a[4]}$ can be expressed in terms of the totally antisymmetric part of $t_{[1]}^{a[3]}$ via (3.79). Note that under $\delta t_{[1]}^{a[3]}=\mathrm{d} \psi_{[0]}{ }^{c[3]}$ and in the gauge where $A_{[3]}=0$, we have that $\psi^{a[3]}=\bar{\psi}^{a[3]}=\partial^{\left[a_{1}\right.} \lambda^{\left.a_{2} a_{3}\right]}$ and therefore $\delta t_{\left[b \mid a_{1} a_{2} a_{3}\right]}=$ 0 , as it should. The field $t_{[1]}^{a[3]}$ plays the role of the connection $\omega_{[3]}^{a[10]}$ introduced in (3.58), upon dualisation of $t_{[1]}^{a[3]}$ on its form index and exchanging the role of form and frame indices. The equations of motion (3.82) imply that (i) $t_{\left[b \mid a_{1} a_{2} a_{3}\right]}=\partial_{[b} C_{\left.a_{1} a_{2} a_{3}\right]}$, thereby re-introducing a three form on-shell, and (ii) the mixed-symmetric part $t_{a_{1} a_{2} a_{3} \| b}=$ $3 \partial_{b} \partial_{\left[a_{1}\right.} \lambda_{\left.a_{2} a_{3}\right]}$. But this is precisely in the form of its residual gauge transformations in the gauge where $A_{[3]}=0$, so that $t_{a[3] \| b}$ is pure gauge and does not carry any local degree of freedom.

Let us demonstrate that the above action makes contact with the unfolded formalism given earlier in this section. Equation (3.83) can be written as

$$
\mathrm{d} e_{[9]}^{a[3]}+h_{b_{1}} \wedge \ldots \wedge h_{b_{7}} \wedge \tilde{\omega}_{[3]}^{a[3] b[7]}=0 .
$$

where

$$
\tilde{\omega}_{[3]}^{a[10]}=-\frac{1}{7 !} h^{a_{1}} h^{a_{2}} h^{a_{3}} F_{c[4]} \epsilon^{a[7] c[4]}
$$

plays the role of the connection appearing in (3.59). More precisely, it is the part of $\omega_{c[3]}{ }^{a[3]} b[7]$ that is antisymmetrised in its ten indices that are written as the two index blocks $c[3]$ and $b[7]$ that appears is (3.59), therefore, we rewrite

$$
\tilde{\omega}_{c[3]}{ }^{a[3]}{ }_{c[7]}=\frac{3 ! 7 !}{10 !} \epsilon_{c[7] f} F^{f a[3]},
$$

while performing the antisymmetrisation over the ten indices $a[10]$ on the right-hand side of (3.89) explicitly gives

$$
\tilde{\omega}_{c[3]}{ }^{a[10]}=\frac{4 !}{10 !} \epsilon^{a[10] b} F_{b c[3]} .
$$

Note that the component of $e_{b[9] \mid a[3]}$ that transforms in the tensor product [10] $\otimes[2]$ of GL(11) is pure gauge on-shell, as can be seen by suitably projecting (3.83) and using (3.87). Thus, it is only the GL(11)-irreducible component $A_{b[9] \| a[3]}$ of $e$ that is glued to the zero-forms on-shell. To repeat, the components $e_{\left[b_{1} \ldots b_{9} \mid b_{10}\right] a_{1} a_{2}}$ are pure gauge on-shell and can therefore be eliminated in a gauge, leaving only the component $A_{b[9] \| a[3]}$ with a differential gauge invariance in terms of the sole GL(11)-irreducible component $\xi_{a[8] \| b[3]}$ : $\delta_{\xi} A_{b[9] \| a[3]}=\partial_{b} \xi_{b[8] \| a[3]}$. In this gauge, the field equations (3.83) then reduce to

$$
\partial_{a} A_{a[9] \|}{ }^{b[3]}=\frac{4 ! 7 !}{10 !} \epsilon_{a[10] c} F^{c b[3]} .
$$


which we recognise as equation (3.50) in the gauge where $\Xi^{a[9] \|_{b[2]}}$ is set to zero. Upon acting with $\partial^{b}$ and antisymmetrising over the four $b$ indices, we get

$$
\partial^{b} \partial_{a} A_{a[9] \|}^{b[3]}=\frac{4 ! 7 !}{10 !} \epsilon_{a[10] c} \partial^{c} \partial^{\left[b_{1}\right.} A^{\left.b_{2} b_{3} b_{4}\right]},
$$

which is nothing but the equation (3.39) up to an inessential coefficient.

\subsection{Higher dualisations on-shell}

In this section we will dualise the higher level components of the representation space $\mathcal{T}$ given in equation (3.1). We consider a generic tensor in the list, say $F_{a[4] \| b(n)}$, and dualise it on all $n$ indices $b$ so as to define

$$
G_{c[10]\|\ldots\| f[10]|| a[4]}^{(n)}:=\epsilon_{c[10] b_{1}} \epsilon_{d[10] b_{2}} \ldots \epsilon_{f[10] b_{n}} F_{a[4] \|} \|^{b(n)} .
$$

Using the symmetries of $F_{a[4] \| b(n)}$ we now show that the $G^{(n)}{ }_{c[10]\|\ldots . \mid f[10]\| a[4]}$ is GL(11) irreducible. Explicitly, we find that

$$
\begin{aligned}
\epsilon^{c[10] f_{1}} G^{(n)}{ }_{c[10]\|\ldots . \mid f[10]\| a[4]} & =(-10 !) \delta_{b_{1}}^{f_{1}} \epsilon_{d[10] b_{2}} \ldots \epsilon_{f_{1} f[9] b_{n}} F_{a[4] \|}{ }^{b(n)} \\
& =10 ! \epsilon_{d[10] b_{2}} \ldots \epsilon_{f[9] b_{1} b_{n}} F_{a[4] \|} b(n) \equiv 0,
\end{aligned}
$$

and

$$
\begin{aligned}
\epsilon^{c[10] a_{1}} G_{c[10]|| \ldots\|f[10]\| a[4]}^{(n)} & =(-10 !) \delta_{b_{1}}^{a_{1}} \epsilon_{d[10] b_{2}} \ldots \epsilon_{f[10] b_{n}} F_{a[4]\|\|^{b(n)}} \\
& =(-10 !) \epsilon_{d[10] b_{2}} \ldots \epsilon_{f[10] b_{n}} F_{b_{1} a[3] \|} \|^{b_{1} b_{2} \ldots b(n)} \equiv 0 .
\end{aligned}
$$

We adopt the short-hand notation in which $G^{(n)}{ }_{c[10]|| \ldots|| f[10] \mid \| a[4]}$ is denoted by $G_{[10, \ldots, 10,4]}^{(n)}$, where the number of columns of height 10 is $n$. This object satisfies the trace conditions

$$
\begin{aligned}
\operatorname{Tr}_{i j}^{10} G_{[10, \ldots, 10,4]}^{(n)}=0, & 1 \leq i<j \leq n \\
\operatorname{Tr}_{i n+1}^{4} G_{[10, \ldots, 10,4]}^{(n)}=0, & 1 \leq i \leq n,
\end{aligned}
$$

where we recall that the symbols $\operatorname{Tr}_{i j}^{n}$ means that one takes an $n$ trace between indices in the $i$ and $j$ th column. To show the first relation we note that

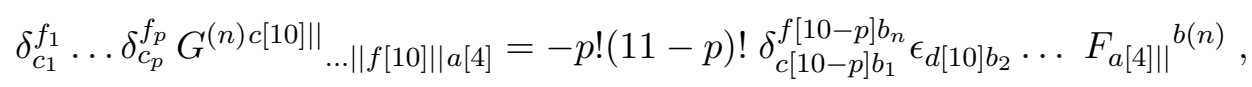

where $\delta_{d[p]}^{c[p]}=\delta^{\left[c_{1}\right.} d_{1} \ldots \delta^{\left.c_{p}\right]} d_{p}$, so that the expression on the left-hand side vanishes only if the antisymmetrised product of Kronecker deltas on the right-hand side of the equation contains $\delta^{b_{n}} b_{n}$, namely only when $p=10$.

To show equation (3.98) we note that

$$
G^{(n) a[p] c[10-p]}\|d[10]\| \ldots\|f[10]\| a[4]=\epsilon^{a[p] c[10-p] b_{1}} \epsilon_{d[10] b_{2}} \ldots \epsilon_{f[10] b_{n}} F_{a[4] \| b_{1}} b_{2} \ldots b_{n}
$$

which only gives zero when $p=4$, i.e. when all the four indices $a$ 's of $F_{a[4] \| b_{1}} b_{2} \ldots b_{n}$ are antisymmetrised with one of the $n$ indices in the set $b(n)$. Obviously, the result is unchanged if one took four traces involving any another of the $n$ columns of length 10 in $G_{[10, \ldots, 10,4]}^{(n)}$. 

that

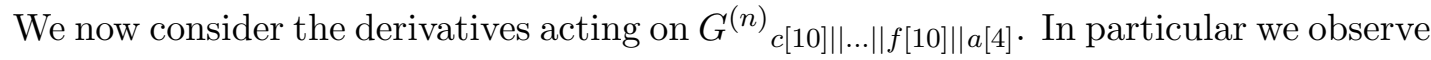

$$
\partial_{\left[a_{1}\right.} G^{(n) c[10]\|d[10]\| \ldots|| f[10]}{ }_{\left.\| a_{2} \ldots a_{5}\right]}=\epsilon^{c[10] b_{1}} \epsilon^{d[10] b_{2}} \ldots \epsilon^{f[10] b_{n}} \partial_{\left[a_{1}\right.} F_{\left.a_{2} \ldots a_{5}\right] \|}{ }^{b(n)}=0,
$$

which is a consequence of the differential equations (3.10) obeyed by the hierarchy of tensors in the set $\mathcal{T}$ in (3.1) together with the GL(11)-irreducible symmetry properties (3.3) of these tensors. Whereupon using the generalised Poincare lemma [31] the GL(11)-irreducible tensors $G_{[10, \ldots, 10,4]}^{(n)}$ can be expressed as generalised curvature tensors of GL(11)-irreducible potentials:

$$
G_{c[10]|| \ldots|| f[10], a[4]}^{(n)}=4(10)^{n} \partial_{c} \ldots \partial_{f} \partial_{a} A^{(n)}{ }_{c[9]|| \ldots .|f[9]| \mid a[3]},
$$

The tensors $G^{(n)}\left(A^{(n)}\right)$ are invariant under the following gauge transformations

$$
\delta_{\lambda} A_{[9, \ldots, 9,3]}^{(n)}=\mathrm{d}^{\{n\}} \lambda_{[9, \ldots, 9,8,3]}^{(n)}+\mathrm{d}^{\{n+1\}} \lambda_{[9, \ldots, 9,2]}^{(n+1)} .
$$

where

$$
\begin{aligned}
\mathrm{d}^{\{n\}} \lambda_{[9, \ldots, 9,8,3]}^{(n)} \rightarrow & 9 \partial_{c_{1}^{n}} \Lambda_{c^{1}[9]|| \ldots\left\|c^{n-1}[9]\right\| c^{n}[8] \| a[3]}+9 x \partial_{c_{1}^{n-1}} \Lambda_{c^{1}[9]|| \ldots\left\|c^{n-1}[8] c_{1}^{n}\right\| c^{n}[8] \| a[3]} \\
& +\ldots+9 x \partial_{c_{1}^{1}} \Lambda_{c^{1}[8] c_{1}^{n}\left\|\ldots . \mid c^{n-1}[9]\right\| c^{n}[8] \| a[3]}, \quad x=-\frac{9}{8} .
\end{aligned}
$$

The first term on the right-hand side of equation (3.87) can be depicted by the Young tableau

$$
\begin{array}{ccc|c|c|}
\cline { 1 - 1 } c_{1} & \ldots & f_{1} & a_{1} \\
\cline { 1 - 1 } c_{2} & \ldots & d_{2} & a_{2} \\
\cline { 1 - 1 } c_{2} & \ldots & f_{2} & a_{2} \\
\cline { 1 - 1 } & \ldots & \vdots & \\
\vdots & \ldots & \vdots & \\
\cline { 1 - 1 } c_{9} & \ldots & c_{9} \\
\hline
\end{array}
$$

Finally, the curvatures $G^{(n)}, n=0,1, \ldots$ are related by

$$
\partial_{g} G_{c[10]\|\ldots\| f[10] \| a[4]}^{(n)}=-\frac{1}{10 !} \epsilon_{g}^{b[10]} G_{b[10]\|c[10]\| \ldots . \mid f[10] \| a[4]}^{(n+1)}
$$

leading to a corresponding duality relation for the first differentials of the various potentials:

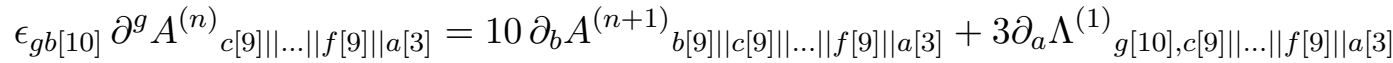

$$
\begin{aligned}
& +Y\left(\partial_{f} \Lambda_{g[10], c[9]|| \ldots|| f[8] \| a[3])}^{(2)}\right.
\end{aligned}
$$

where the symbol $Y$ means the projection of the $9 n+3$ indices $\{c[9]\|\ldots\| f[9] \| a[3]\}$ on the GL(11) Young tableau with $n$ columns of height 9 and one of height 3 . Drawing from the experience we gained from the frame-like formulation of the gauge field $A_{[9,3]}$, we expect that the first-order duality relation (3.107) become free of inhomogeneous term when expressed in terms of the frame-like frame fields and connections. 


\section{Discussion}

In this paper we have shown how the manifestly Lorentz and gauge covariant formulation of the irreducible representations of the Poincaré group leads naturally to a description of the dynamics of the massless point particle in terms of an infinite number of gauge fields which obey first order duality relations. Gauge fields of this type were automatically contained within the $E_{11}$ non-linear realisation which is conjectured to be a symmetry of the underlying theory of strings and branes. The $E_{11}$ symmetry acts on the infinite number of gauge fields rotating them into each other and this part of the symmetry can be thought of as an extension of what we usually regard as a duality symmetry.

Duality symmetries have played an important part in theoretical physics and one may hope that the extension of the symmetry given in this paper may prove useful in future work. Certainly it will act as a very useful guide when formulating the equations of motion that follow from the $E_{11}$ non-linear realisation. The precise way the duality relations found here are realised in the $E_{11}$ approach will be published in a future paper.

An important aspect of the present paper is that the whole analysis concerns massless gauge fields freely propagating in Minkowski background. We expect important conceptual changes as the AdS background is considered instead. Indeed, in AdS gauge fields are characterised by mass terms that all degenerate to zero in the flat limit. As a matter of fact, manifest electric-magnetic duality-invariant unfolded equations for massless fields freely propagating in $A d S_{4}$ background have been given in [39]. In this formulation, the rigid group of transformation $\operatorname{Sp}(8, \mathbb{R})$ preserves the unfolded equations, since the left-hand side of the field equations transforms covariantly with respect to this group. As discussed in [39], the $\operatorname{Sp}(8, \mathbb{R})$ covariant formulation experiences a degeneracy when the flat limit is considered, thereby indicating that electric-magnetic duality acts very differently depending on the curvature of the background. A very appealing aspect of the unfolded formulation for massless gauge fields in $A d S_{4}$ presented in [39] is that a $u(1)$ subalgebra of $s p(8, \mathbb{R})$ acts on the fields as the electric-magnetic transformation, through an explicit doubling of the set of fields related by the action of the $u(1)$ symmetry. This feature is linked to the dimensionality of $A d S_{4}$ and the use of appropriate spinor variables. It would be very interesting to investigate whether such a manifest duality symmetry could be extended to unfolded systems in higher spacetime dimensions.

Finally, it would also be interesting to see if the duality relations found in this paper could be extended to the Yang-Mills case. A discussion of the usual electro-magnetic duality transformations in the context of Yang-Mills theory was given in [40].

\section{Acknowledgments}

PW wishes to thank Paul Cook for discussions and the SFTC for support from Consolidated grant number ST/J002798/1. NB and PS want to thank M. A. Vasiliev for discussions about electric-magnetic duality in unfolded formulations. NB is Research Associate of the Fonds de la Recherche Scientifique-FNRS (Belgium). His work was partially supported by a contract "Actions de Recherche concertées -Communauté française de Belgique" AUWB- 
2010-10/15-UMONS-1. NB thanks the Mathematics Department of King's College London for hospitality, while PS and PW thank the hospitality of the Service de Mécanique et Gravitation of UMONS where parts of this work were done. PS and PW acknowledge the support of the Conicyt grant DPI 20140115.

\section{A Notation}

In this appendix we give some of the notation used in this paper. While these definitions are given in the text it may not always be easy for the reader to find them and so we collect them here for easy reference. The first few sections of the paper are written without using elaborate notation so that the reader can get used to the subject, but as the paper progresses we need more and more indices and so we introduce a shorthand notation.

We separate blocks of antisymmetrised or symmetrised indices on the fields by putting a double bar, for example $A_{a_{1} a_{2} a_{3} \| b_{1} \ldots a_{9}}$. We eventually use a shorthand for blocks of antisymmetric and symmetric indices by denoting $A_{a[n]} \equiv A_{\left[a_{1} \ldots a_{n}\right]} \equiv A_{a_{1} \ldots a_{n}}$ for blocks of antisymmetric indices and $S_{a(n)} \equiv S_{\left(a_{1} \ldots a_{n}\right)} \equiv S_{a_{1} \ldots a_{n}}$ for symmetrised indices. We use the strength-one (anti)symmetrisation convention.

In the early sections of the paper we denote antisymmetrisation in the usual way that is $F_{a_{1} a_{2} a_{3} a_{4}}=4 \partial_{\left[a_{1}\right.} A_{\left.a_{2} a_{3} a_{4}\right]}$. However, once we have more indices to cope with we adopt the convention that when an index with the same Latin label occurs in the same up, or down, position in an equation, it is automatically antisymmetrised. For example, when we write $F_{a[4]}=4 \partial_{a} A_{a[3]}$ we automatically mean $F_{a_{1} a_{2} a_{3} a_{4}}=4 \partial_{\left[a_{1}\right.} A_{\left.a_{2} a_{3} a_{4}\right]}$.

When we are discussing forms we label the degree of the form by a number in square brackets written as a subscript, for example $A_{[3]}=\frac{1}{3 !} d x^{a_{1}} \wedge d x^{a_{2}} \wedge d x^{a_{3}} A_{a_{1} a_{2} a_{3}}$.

Open Access. This article is distributed under the terms of the Creative Commons Attribution License (CC-BY 4.0), which permits any use, distribution and reproduction in any medium, provided the original author(s) and source are credited.

\section{References}

[1] P.A.M. Dirac, Quantized Singularities in the Electromagnetic Field, Proc. Roy. Soc. Lond. A 133 (1931) 60 [INSPIRE].

[2] G. 't Hooft, Magnetic Monopoles in Unified Gauge Theories, Nucl. Phys. B 79 (1974) 276 [INSPIRE].

[3] A.M. Polyakov, Particle Spectrum in the Quantum Field Theory, JETP Lett. 20 (1974) 194 [INSPIRE].

[4] C. Montonen and D.I. Olive, Magnetic Monopoles as Gauge Particles?, Phys. Lett. B 72 (1977) 117 [INSPIRE].

[5] E. Cremmer and B. Julia, The $N=8$ Supergravity Theory. 1. The Lagrangian, Phys. Lett. B 80 (1978) 48 [INSPIRE].

[6] M.K. Gaillard and B. Zumino, Duality Rotations for Interacting Fields, Nucl. Phys. B 193 (1981) 221 [InSPIRE]. 
[7] P.C. West, $E_{11}$ and M-theory, Class. Quant. Grav. 18 (2001) 4443 [hep-th/0104081] [INSPIRE].

[8] T. Curtright, Generalized Gauge Fields, Phys. Lett. B 165 (1985) 304 [inSPIRE].

[9] C.M. Hull, Strongly coupled gravity and duality, Nucl. Phys. B 583 (2000) 237 [hep-th/0004195] [INSPIRE].

[10] N. Boulanger, S. Cnockaert and M. Henneaux, A note on spin s duality, JHEP 06 (2003) 060 [hep-th/0306023] [INSPIRE].

[11] P. West, Introduction to Strings and Branes, Cambridge University Press (2012), chapter 17.

[12] F. Riccioni and P.C. West, The $E_{11}$ origin of all maximal supergravities, JHEP 07 (2007) 063 [arXiv:0705.0752] [INSPIRE].

[13] E.A. Bergshoeff, I. De Baetselier and T.A. Nutma, $E_{11}$ and the embedding tensor, JHEP 09 (2007) 047 [arXiv: 0705.1304] [INSPIRE].

[14] F. Riccioni and P.C. West, Dual fields and $E_{11}$, Phys. Lett. B 645 (2007) 286 [hep-th/0612001] [INSPIRE].

[15] F. Riccioni, D. Steele and P.C. West, Duality Symmetries and $G^{+++}$Theories, Class. Quant. Grav. 25 (2008) 045012 [arXiv:0706.3659] [INSPIRE].

[16] N. Boulanger, P.P. Cook and D. Ponomarev, Off-Shell Hodge Dualities in Linearised Gravity and E11, JHEP 09 (2012) 089 [arXiv: 1205.2277] [INSPIRE].

[17] E.P. Wigner, On Unitary Representations of the Inhomogeneous Lorentz Group, Annals Math. 40 (1939) 149 [Nucl. Phys. Proc. Suppl. 6 (1989) 9].

[18] V. Bargmann and E.P. Wigner, Group Theoretical Discussion of Relativistic Wave Equations, Proc. Nat. Acad. Sci. 34 (1948) 211 [inSPIRE].

[19] X. Bekaert and N. Boulanger, Mixed symmetry gauge fields in a flat background, hep-th/0310209 [INSPIRE].

[20] X. Bekaert and N. Boulanger, Tensor gauge fields in arbitrary representations of $\mathrm{GL}(D, R)$. II. Quadratic actions, Commun. Math. Phys. 271 (2007) 723 [hep-th/0606198] [INSPIRE].

[21] X. Bekaert and N. Boulanger, The unitary representations of the Poincaré group in any spacetime dimension, hep-th/0611263 [INSPIRE].

[22] M.A. Vasiliev, Cubic interactions of bosonic higher spin gauge fields in $A d S_{5}$, Nucl. Phys. B 616 (2001) 106 [Erratum ibid. B 652 (2003) 407] [hep-th/0106200] [INSPIRE].

[23] M.A. Vasiliev, Actions, charges and off-shell fields in the unfolded dynamics approach, Int. J. Geom. Meth. Mod. Phys. 3 (2006) 37 [hep-th/0504090] [InSPIRE].

[24] E.D. Skvortsov, Mixed-Symmetry Massless Fields in Minkowski space Unfolded, JHEP 07 (2008) 004 [arXiv:0801.2268] [INSPIRE].

[25] K.B. Alkalaev, M. Grigoriev and I. Yu. Tipunin, Massless Poincaré modules and gauge invariant equations, Nucl. Phys. B 823 (2009) 509 [arXiv:0811.3999] [InSPIRE].

[26] N. Boulanger, C. Iazeolla and P. Sundell, Unfolding Mixed-Symmetry Fields in AdS and the BMV Conjecture: I. General Formalism, JHEP 07 (2009) 013 [arXiv:0812.3615] [INSPIRE].

[27] W. Siegel and B. Zwiebach, Gauge String Fields from the Light Cone, Nucl. Phys. B 282 (1987) 125 [inSPIRE]. 
[28] W. Siegel, Fields, hep-th/9912205 [InSPIRE].

[29] M. Hamermesh, Group theory and its application to physical problems, Dover, New York, U.S.A. (1989).

[30] W. Fulton and J. Harris, Representation Theory: A First Course, Corrected edition, Graduate Texts in Mathematics/Readings in Mathematics, Springer (1991).

[31] X. Bekaert and N. Boulanger, Tensor gauge fields in arbitrary representations of GL $(D, R)$ : Duality and Poincaré lemma, Commun. Math. Phys. 245 (2004) 27 [hep-th/0208058] [INSPIRE].

[32] X. Bekaert, N. Boulanger and D. Francia, Mixed-symmetry multiplets and higher-spin curvatures, J. Phys. A 48 (2015) 225401 [arXiv:1501.02462] [INSPIRE].

[33] P.C. West, Very extended $E_{8}$ and $A_{8}$ at low levels, gravity and supergravity, Class. Quant. Grav. 20 (2003) 2393 [hep-th/0212291] [INSPIRE].

[34] H. Nicolai, P.K. Townsend and P. van Nieuwenhuizen, Comments On Eleven-Dimensional Supergravity, Lett. Nuovo Cim. 30 (1981) 315 [INSPIRE].

[35] I.A. Bandos, N. Berkovits and D.P. Sorokin, Duality symmetric eleven-dimensional supergravity and its coupling to M-branes, Nucl. Phys. B 522 (1998) 214 [hep-th/9711055] [INSPIRE].

[36] N. Boulanger and D. Ponomarev, Frame-like off-shell dualisation for mixed-symmetry gauge fields, J. Phys. A 46 (2013) 214014 [arXiv:1206.2052] [InSPIRE].

[37] O.A. Gelfond and M.A. Vasiliev, Higher-Rank Fields and Currents, arXiv:1312.6673 [INSPIRE].

[38] P.C. West, Very extended $E_{8}$ and $A_{8}$ at low levels, gravity and supergravity, Class. Quant. Grav. 20 (2003) 2393 [hep-th/0212291] [INSPIRE].

[39] M.A. Vasiliev, On Conformal, $\mathrm{SL}(4, \mathbb{R})$ and $\mathrm{Sp}(8, \mathbb{R})$ Symmetries of $4 d$ Massless Fields, Nucl. Phys. B 793 (2008) 469 [arXiv:0707.1085] [InSPIRE].

[40] S. Deser and C. Teitelboim, Duality Transformations of Abelian and Nonabelian Gauge Fields, Phys. Rev. D 13 (1976) 1592 [inSPIRE]. 